\title{
VIth International Symposium on Parkinson's Disease VIe Symposium international sur la maladie de Parkinson
}

\author{
Québec, Qué. Canada. \\ September 24-27, 1978
}

\author{
SPONSORED BY - SOUS L'ÉGIDE DE \\ The Parkinson Foundation of Canada \\ The American Parkinson Disease Association \\ Le Conseil de la recherche en santé du Québec (CRSQ) \\ Le Conseil de recherches médicales du Canada (MRC) \\ World Federation of Neurology
}

PRESIDENT: Dr. Louis J. Poirier

$\begin{array}{lll}\text { ORGANIZING COMMITTEE COMITÉ D'ORGANISATION } \\ \text { Paul Bédard } & \text { Michel Filion } & \text { André Parent } \\ \text { René Boucher } & \text { Pierre Langelier } & \text { Liliane Poirier } \\ \text { Marc Colonnier } & \text { Louis Larochelle } & \text { Jacques Tremblay }\end{array}$

Bromocriptine Associated with a Peripheral

Dopamine Blocking Agent in the

Treatment of Parkinsonism

Y.Agid, P. Pollak, A. M. Bonnet, F. Lhermitte, Clinique de Neurologie et Neuropsychologie, Hôpital de la Salpêtrière, Paris, France

The therapeutic efficacy of Bromocriptine, a drug acting upon dopaminergic receptors, is limited by adverse side effects. The present investigation describes a way to prevent these peripheral adverse reactions by the addition of a peripheral dopaminergic blocking agent (Domperidone), thus allowing administration of higher doses of Bromocriptine. Domperidone $(60 \mathrm{mg}$ daily) or placebo were administred in a double blind study conducted in 17 parkinsonian patients each receiving increasing doses of Bromocriptine. Combined treatment caused a mean significant $79 \%$ reduction in total disability in 8 patients receiving a mean dose of $148 \mathrm{mg}$ of Bromocriptine daily. Vomiting did not occur; involuntary movements and psychic disturbances were similar in nature to those following administration of L-DOPA. In nine patients taking placebo in place of Domperidone, the mean daily dose of Bromocriptine could not be raised beyond $92 \mathrm{mg}$. The mean total disability score in this group was reduced by only $48 \%$. Thus, peripheral blockade of dopamine receptors appears to be a promising tool in the treatment of Parkinsonism with central dopaminergic receptor stimulating agents.

Interet de L'etude de L'Activite Renine Plasmatique (ARP) Chez les Parkinoniens

Allain, H., -Van Den Driessche, J., Sabouraud, O., Laboratorie de Pharmacologie, Faculté de Médecine - Rennes - 35000 - France

Une première étude chez 42 patients parkinsoniens nous apprend que les chiffres d'ARP des non traités ne sont pas différents de ceux du groupe témoin. Chez les parkinsoniens traités sans effets secondaires par la DOPA + IDC les chiffres d'ARP s'effondrent conformément aux données expérimentales. A l'inverse chez les patients traités par la L.DOPA + IDC mais présentant des dyskinésies induites les chiffres d'ARP s'élèvent nettement. Il existe une corrélation étroite entre l'intensité des dyskinésies et cette

The printing of these abstracts was made possible by the generosity of the Parkinson Foundation of Canada. augmentation de l'ARP. Une étude cinétique chez 25 patients, portant sur une heure et demie après la prise unique orale d'un agoniste dopaminergique (L.DOPA + IDC; BROMOCRYPTINE; dérivé de l'Apomorphine) confirment les premiers résultats, la variation de l'ARP étant la plus nette $1 / 2$ heure après la prise du médicament. Aucune corrélation n'a pu être établie entre ARP et chiffres tensionnels. Néanmoins, tous les patients dyskinétiques présentent des tensions artérielles fluctuantes avec poussées, phénomène sans doute dépendant du dysfonctionnement du systéme rénine angiotensine. L'ARP peut donc être considéré comme un index de l'action pharmacologique centrale de la L.DOPA, ainsi qu'un moyen d'approche de la physiopat hologie des dyskinésies induites par la L.DOPA.

The Significance of the Neostriatum and the Nucleus Accumbens for the Rigidity and the Akinesia Induced by Neuroleptic Drugs.

N. - E. Andén, Department of Pharmacology, University of Götborg, S-400 33 Götborg, Sweden.

Local injections of dopamine or apomorphine into the nucleus accumbens or the neostriatum were used in order to investigate the functional role of these nuclei for the motor activity and for the muscle tone of rats. The motor activity of reserpine-treated rats was stimulated to more than normal values following bilateral application of dopamine or apomorphine to the nucleus accumbens. This effect was blocked by systemic treatment with neuroleptic drugs such as pimozide. No increase in motor activity was produced by apomorphine given into the neostriatum. The muscle tone of rats was recorded mechanographically at passive dorsiflexion of one hindfoot. Reserpine increased the muscle tone. This reserpine-induced rigidity was completely inhibited following bilateral injections of apomorphine into the neostriatum, but not into the nucleus accumbens. The effect of apomorphine on the muscle tone was completely antagonized by systemic treatment with haloperidol. Stereotypies such as gnawing and licking were observed following the injections of apomorphine into the neostriatum, but not into the nucleus accumbens. The results indicate that the neuroleptic-induced akinesia and rigidity are caused by a lack of dopamine influence in the nucleus accumbens and in the neostriatum, respectively.

Presynaptic and Postsynaptic Effects of Dopamine Receptor Blocking Agents.

N.-E. Andén and M. Grabowska-Andén, Department of Pharmacology, University of Göteborg, S-400 33 Göteborg, Sweden.

The relative effects of haloperidol, pimozide, R 28935 and clozapine on postsynaptic and presynaptic dopamine receptors in the rat corpus striatum were evaluated by their abilities to inhibit changes induced by apomorphine, $2 \mathrm{mg} / \mathrm{kg}$ i.p. The postsynaptic effects were measured by means of the apomorphine-induced rotation to the $\mathrm{KCl}$-treated side of reserpinepretreated rats in which $1 \mu 125 \% \mathrm{KCl}$ was injected into the corpus striatum on one side. The presynaptic effects were measured by means of the apomorphine-induced decrease in the DOPA accumulation following DOPA decarboxylase inhibition in combination with interruption of the nerve impulse flow by $\gamma$-butyrolactone. The postsynaptic and the presynaptic effects of apomorphine were inhibited by about the same doses of haloperidol as well as pimozide. R 28935 was about 100 times more potent in blocking the postsynaptic than the presynaptic actions whereas clozapine was about 30 times more effective on the postsynaptic receptors. A preferential blockade of postsynaptic dopamine receptors might reduce the risk of tardive dyskinesia. 


\section{Neuron Identification in the Neostriatum by Means of Retrograde Transport of Herpes Simplex Virus.}

I.J.Bak, C. H. Markham, M. L.Cook and J.G.Stevens, Department of Neurology, Reed Neurological Research Center, UCLA School of Medicine, Los Angeles, California 90024, U.S.A.

A neurotropic virus, Herpes simplex virus (HSV) type 1 was used as a tracer to study strio-nigral neurons. 1 ul of highly concentrated HSV $(10,000-40,000$ PFU) was inoculated into the left substantia nigra. At 4 to 5 days the animals were sacrificed for light and electron microscopy and another group was prepared for indirect immunoperoxidase histochemistry for HSV infected neurons. On the 5th day the ipsilateral striatum showed a large number of virus infected neurons whereas no virus infected cells were seen in the contralateral neostriatum or in either side of the frontal cortex. Indirect immunoperoxidase histochemistry verified this distribution.

In the striatum, neurons show a loss of nucleolus, pale nucleoplasm and due to the migration of chromatin to the nuclear membrane, the nuclear membrane appeared more distinct. Under the electron microscope such abnormal cells were seen to contain virus particles, both nucleocapsids (immature virus) and virions (mature virus). The HSV infected cells were mostly ovoidal medium sized neurons measuring 15 to $20 \mathrm{um}$ in dia meter. A few of the rare large neurons were infected.

Light microscopic evaluation of the semi-thin sections revealed the percentages of infected cells to range from 14 to $47 \%$. A large number of these infected cells were observed in the head of the neostriatum.

Present results show that this virus labelling technique allows ultrastructural characterization of strionigral neurons and thereby differentiate them from other neuronal populations.

Newer Therapeutic Approaches in Extrapyramidal Disorders

A. Barbeau and M. Roy, Clinical Research Institute of Montreal, Montreal, Quebec, Canada, H2W IR7.

This paper will present a review of four newer therapeutic approaches to basal ganglia dysfunction, as utilized in our own clinic over the last four years: (1) Dopamine Agonists with or without Levodopa in Parkinson's disease. We have tested, for this purpose Piribedil, Nomifensin and Bromocriptine. These drugs are useful for a finite period of benefit, for a minority of parkinsonians with mild disabilities of recent onset. None of these drugs provided an advantage in combination with Levodopa. (2) Diprobutine (LCG-21519), this drug has definite antiparkinsonian activity in man, but the margin of safety between therapeutic efficacy and serious side-effects is very small. (3) Pro-Leu-Gly- $\mathrm{NH}_{2}$ (PLG), a tripeptide shown to be very effective in potentiating Levodopa's antiakinesia effect. (4) Choline and Lecithin, acetylcholine precursors who are of marked efficacy against neuroleptic induced tardive dyskinesias and to a lesser extent against chorea.

\section{Gaba and Dopamine Neurons: Relation to Extrapyramidal Disorders} G. Bartholini, B. Scatton, P. Worms

B.Zivkovic, K.G. Lloyd and R. Tissot*, Research Dept., Synthelabo-L.E.R.S., 58, rue de la Glacière, 75013 Paris, France and *University Psychiatric Clinic, Geneva, Switzerland.

In parkinsonian patients the inhibitory GABA influence on nigro-striatal dopamine neurons is descreased as indicated by the reduction of glutamic acid decarboxylase in substantia nigra and striatum. This reduction probably reflects an homeostatic mechanism allowing an enhanced firing rate of the still present dopamine neurons. In patients treated with LDOPA, this enhanced firing possibly causes and exaggerated release of dopamine leading to involuntary movements. This provides the rationale for the treatment of L-DOPA-Induced involuntary movements by enhancing GABA-ergic transmission. SL 76002 is a new, nontoxic, GABA mimetic agent as indicated by pharmacological and biochemical data. This compound reduces the dopamine turnover in striatum and its action is more pronounced after activation of the firing of the dopaminergic neurons. Thus, SL 76002 is expected to decrease the L-DOPA-induced involuntary movements. In this regard, clinical data from parkinsonian patients will be discussed.
Westphal Phenomenon in Dystonia.

N. Bathien, S. Tomas and P. Rondot, Service de Neurologie, Centre Hospitalier Sainte-Anne, Paris, France.

EMG activity in tibialis anterior muscle (TA) and in soleus muscle (SOL) was recorded in 5 patients with dystonia: 3 dystonia musculorum deformans, 1 post therapeutic irradiation hemiathetosis and 1 athetosis. Passive dorsal flexion of the ankle evoked a tonic E.M.G. activity in TA during all the shortening of the muscle. This response to muscle shortening was called Westphal phenomenon (W.P.) or shortening reaction. W.P. was phasic in some normal subject muscles and was only recorded during the dynamic phase of muscle shortening (KATZ and RONDOT, 1978). A linear relationship was found between EMG activity in TA and the joint angle, and a threshold angle; but W.P. was independent of shortening velocity. W.P. in dystonia was compared to W.P. in Parkinsonian rigidity and spasticity. Infiltration of the ankle-joint with procaine abolished W.P. in TA muscle. The effect of joint afferents in W.P. is discussed.

\section{EMG Activity and Spinal Reflexes in L. Dopa Dyskinesia}

N. Bathien, S. Toma and P. Rondot, Service de Neurologie, Centre Hospitalier Sainte-Anne, Paris, France.

Neurophysiologic investigations were performed in 11 Parkinsonian patients with L.DOPA dyskinesia. EMG activity during abnormal movements, monosynaptic reflex ( $\mathrm{H}$ reflex), cutaneous polysynaptic reflex of the lower limb, muscular response to passive movement (shortening and myotatic reaction) were studied. EMG activity during L.DOPA dyskinesia can be classified into 2 types: 1) Choreoid Pattern (Ch.P) was irregular in amplitude and frequency. EMG activity in Flexor and Extensor muscle was not coordinated. 2) Rhythmic Pattern (R H.P) was included with bursts of $500 \mathrm{~ms}$ duration with a frequency of $1-2 \mathrm{c} / \mathrm{s}$. They were synchronous with EMG activity in antagonist muscles. Dopaminergic drugs (Apomorphine and Piribedil) injections (IV) changed Ch.P. to Rh.P. During the hyperkinetic phase, $\mathrm{H} / \mathrm{M}$ ration was increased, the nociceptive polysynaptic reflex was enhanced and the shortening reaction was abolished. No relationship was found between EMG patterns, changes in spinal reflexes, and the clinical types of L.DOPA dyskinesia. These data obtained in man have shown that L.DOPA dyskinesia is due to dopaminergic receptor stimulation (effect of drug injections) and changes in interneuron activity at segmental level.

\section{Essential Tremor and Dystonic Syndromes.}

D. W. Baxter, S. Lal and M. Rasminsky, Division of Neurology, Montreal General Hospital, Montreal, Quebec, H3G IA4.

Essential tremor is generally regarded as a monosymptomatic disorder. However sporadic reports have appeared over the past century suggesting that dystonia and other neurologic symptoms may be meaningfully related to essential tremor. Such an association might have significant implications concerning a central pathogenesis of essential tremor

Several observations emerged when case records of patients presenting with spasmodic torticollis were reviewed. A tremor involving the head and/or hands was not infrequently noted. Some patients had presented to physicians several years earlier complaining of tremor. A strong family history of hand or head tremor was not uncommon.

With these observations in mind the case records of 100 patients who had presented because of essential tremor were retrospectively reviewed. Dystonic symptomatology of some degree was noted in $10^{\circ} \circ$. Dystonia most freqently involved the neck and less commonly involved one upper extremity.

\section{Estrogens, Progesterone and the Extrapyramidal System.}

P. J. Bèdard, J. Dankova, R. Boucher and P. Langelier. Laboratoires de neurobiologie, Hôpital de l'Enfant-Jésus, Québec, Qué. Canada. GIJ 5B3

Estrogens exert a direct inhibitory action on the hypophyseal dopamine receptor responsible for controlling prolactin secretion (Labrie et al in Progress in Prolactin Physiology and Pathology; C. Robyn and M. Hartr eds. Elsevier North Holland 121-136.) We have demonstrated a beneficial effect of estrogens in patients sulfering from L-DOPA-induced dyskinesias 
or from neuroleptic induced tardive dyskinesias. This suggests that estrogens have an antidopaminergic action also in the striatum. In a group of twenty-five female rats, a radiofrequency lesion was performed stereotaxically in the left entopeduncular nucleus. Circling (turns per minute) and general motility (with a motility box) were then tested several times after apomorphine $0.5 \mathrm{mg}$ s.c. We then divided the animals into four groups. They were kept in constant light conditions and treated twice a day for seven days with one of the following (A) 17-B estradiol, 2 ug s.c. (B) R-5020 a potent progestative compound, 200 ug s.c. (C) a combination of $A$ and $B$ (D) vehicle. During the period of hormone administration, the animals were tested three times for circling and motility after apomorphine. They were then castrated, allowed to recover and the same procedure repeated. Estradiol causes a significant $(50 \%)$ reduction in apomorphine-induced motility, especially after castration. Apomorphine induced circling, however. was reduced as efficiently $(40 \%)$ by $R-5020$ as by estradiol and was not modified by castration. Our results suggest that estrogens and perhaps profesterone affect dopaminergic transmission not only in the hypothalamico-pituitary axis but also in the striopallidal system. The exact mechanism is currently under investigation. Clinical applications are obvious.

\section{New Approaches to the Management of Drug-Induced Involuntary Movements. \\ P. Bedard, P. Price, D. Parkes and C. D. Marsden, \\ University Department of Neurology, Institute of Psychiatry and King's College Hospital Medical School, Denmark Hill, London S.E.5., England.}

Involuntary movements are now the commonest dose-limiting side effect of levodopa treatment of Parkinson's disease. Such levodopa-induced dyskinesias can be reversed by either decreasing the dose of levodopa or by adding a typical neuroleptic dopamine antagonist such as phenothiazine or butyrophenone. However, both decrease dyskinesias at the expense of increased akinesia and rigidity.

Studies in animals have suggested that some atypical neuroleptics may inhibit dyskinesias more specifically. We report clinical studies of the use of oxiperomide and tiapride in patients with Parkinson's disease treated with levodopa or bromocriptine.

\section{Stereotactic Targets for Dystonias and Dyskinesias and Their Relationship to Cortico-Bulbar Fibers. \\ C. Bertrand, P. Molina-Negro, S. N. Martinez,}

Hôpital Notre-Dame, Montréal, H2L 1 M4

Using proportional measurements the thalamic target for dyskinesias, has been established more accurately than was thought possible in view of individual variations. The target for dystonias situated more anteriorly in posterior VOI gives very commendable results. In both instances relationship to adjoining functional structures has been determined; with the help of stimulation and recording spino-thalamic and classical cortico-spinal fibers as well as the sub-thala mic nucleus can be readily avoided. However. cortico-bulbar and adjacent corticospinal fibers lie immediately lateral to and in the same coronal plane as the target for dystonias. As reported previously, bilateral lesions at that level produced cortico-bulbar manifestations in $10 \%$ of cases compared with $2 \%$ for overall thalamic lesions and this has been a major deterrent. Safety can be increased considerably using unilateral, more posterior medical hemispheric lesions together with physiologic safeguards. Cortico-bulbar symptoms and their similarity to athetosis are discussed.

\section{Principles and Problems of Therapy.}

W. Birkmayer, L. Boltzmann,

Institute for Neuro-chemistry, Vienna, Austria.

The main problem in the treatment of Parkinson's syndrom is the substitution of the insufficient transmitters. The second problem is the inhibition of side-effects caused by the loss of the biochemical balance. Ten years experience will be presented of the combined medication of L'Dopa plus inhibitors of Decarboxylase, plus L'Tryptophan, plus Amitriptyline and plus L'Deprenil. The aim is to reach a balanced storage of various transmitters.

\section{Cholinesterases in Brain, Ganaglionic and Neuromuscular Synapses. M. Brzin, Institute of Pathophysiology, School of Medicine, University of Ljubljana, 61105 Yugoslavia}

Using thiocyanate modification of $\mathrm{Cu}$-thiocholine procedure, ultrastructural localization of cholinesterases was studied in cells of various brain regions, sympathetic ganglia and in neuromuscular junctions. In neurones ChE-s activity is predominantly localized intracellularly in the endoplasmic reticulum and at the axolemma of presynaptic axons. The synaptic regions (synaptic membranes and the cleft) do not exhibit a pronounced $\mathrm{ChE}$ activity. In the neuromuscular junction ChE activity is highly condensed in the junctional region. $\mathrm{AChE}$ is the predominant $\mathrm{ChE}$ found in neuronal tissue and the muscle of the rat. The proportion of BuChE activity relative to $\mathrm{AChE}$ varies depending on the tissue, whereas the ultrastructural localization of the enzyme closely resembles the localization of AChE. Cytochemical observations about the localization of ChE-s in various structures are in agreement with quantitative data obtained by microgasometry on isolated single neurones and motor endplates, suggesting that in the latter $\mathrm{ChE}$ activity excedes by far the activity of in neurone to neurone synapses. Cytochemical approaches for ultrastructural localization of cholinesterases and possible significance of differences in localization of the enzyme in cholinergic synapses will be discussed.

Pars Compacta of the Substantia Nigra Modulates Locomotion and
Circling but is not Involved in Regulating Food and Water Intake.

L. L. Butcher ' and G. K. Hodge, 2 Department of Psychology 1, 2 and Brain Research Institute', University of California.

Los Angeles, CA, 90024, U.S.A.

Taking advantage of the anatomic orientation of pars compacta (PC) of the substantia nigra, we have made radiofrequency lesions in the PC that are more selective than those produced by other technics, including ablations deriving from intranigral infusion of various cytotoxins (e.g., 6hydroxydopamine). Precise unilateral lesions of PC produced contralateral circling that peaked $24-48$ hrs after surgery and lasted for 5 days. This behavior was correlated with extensive but not total loss of histochemically assessed dopamine in the caudate-putamen complex; little or no fluorescence decrements were seen in nucleus accumbens and other forebrain catecholamine projection areas. More intense and sustained spontaneous turning was observed after lesions in the reticular formation adjacent to nucleus centralis superior and including portions of that raphe structure; this circling was not correlated with damage to ascending 5-hydroxytryptamine systems, however. Neither $1.5 \mathrm{mg} / \mathrm{kg}$ amphetamine nor 1 $\mathrm{mg} / \mathrm{kg}$ apomorphine elicited turning in PC-lesioned animals after their behavior returned to baselıne levels.

Bilateral radio-frequency lesions confined to PC resulted in the immediate and sustained appearance of hyperactivity but did not alter food or water intake. Both these results and the findings from unilateral PC ablations suggest that nigral dopamine is concerned with motor functions, perhaps exerting a modulatory influence on them, but that (1) its role in motivational processes is minimal, perhaps non-existent, and (2) its involvement in circling behavior is less significant than previously believed. (This research was supported by USPHS grant NS-10928 to L.L.B.)

'Present address: Department of Psychology, University of New Mexico. Albuquerque, NM, 87131, U.S.A.

Dopaminergic, Serotonergic, and Cholinergic Drugs and Their Effects on Levels and Turnover of Neostriatal Acetylcholine ( $\mathrm{ACh}$ ) and Choline (Ch).

Sherrel H. Butcher, Department of Pharmacology and Mental Retardation Center. University of California, Los Angeles, CA, 90024 U.S.A.

Endogenous levels and synthesis of $\mathrm{ACh}$ and $\mathrm{Ch}$ were determined mass spectrometrically following an i.v. injection of $20 \mu \mathrm{mol} k \mathrm{~kg}{ }^{2} \mathrm{H}_{4}-\mathrm{Ch} . \mathrm{L}-$ DOPA, amphetamine, and $\rho-\mathrm{Cl}$-amphetamine increased neostriatal $\mathrm{ACh}$ levels while ${ }^{2} \mathrm{H}_{4}-\mathrm{ACh}$ and ${ }^{2} \mathrm{H}_{4}-\mathrm{Ch}$ were reduced (Table). 5-hydroxytryptophan (5-HTP), haloperidol, pımozide, and atropine reduced caudateputamen ACh levels, whereas oxotremorine had no effect on endogenous content but did decrease ${ }^{2} \mathrm{H}_{4}-\mathrm{ACh}$ (Table). (USPHS NS-10928) 


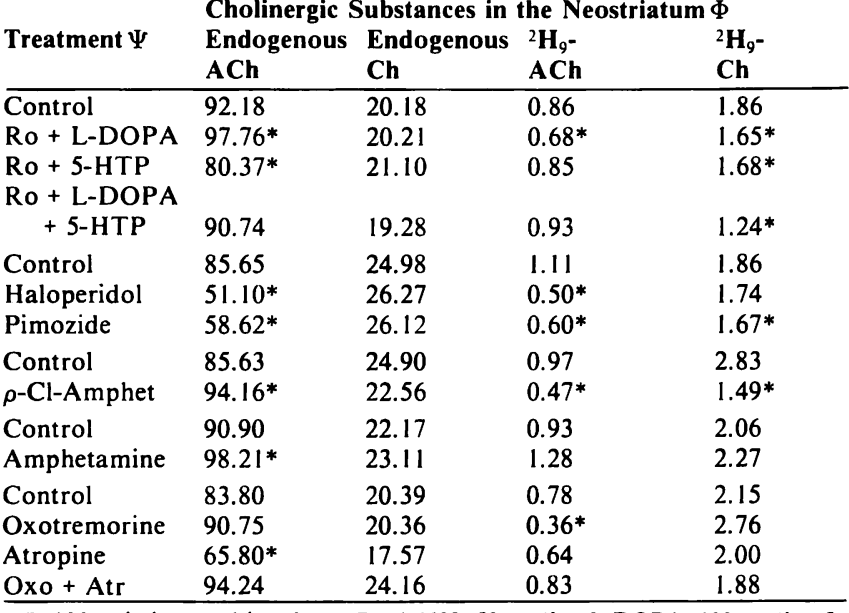

$\Psi$ Abbreviations and i.p. doses; Ro-4-4602, $50 \mathrm{mg} / \mathrm{kg}$; L-DOPA, $100 \mathrm{mg} / \mathrm{kg}$; 5 HTP, $100 \mathrm{mg} / \mathrm{kg}$; Haloperidol, I mg/kg; Pimozide, I mg/kg; p-Cl-Amphetamine, 5 $\mathrm{mg} / \mathrm{kg}$ : Amphetamine, $1 \mathrm{mg} / \mathrm{kg}$; Oxotremorine, $0.3 \mathrm{mg} / \mathrm{kg}$; Atropine, $1 \mathrm{mg} / \mathrm{kg}$.

$\Phi$ Values expressed in $\mathrm{nmol} / \mathrm{g}$ caudate-putamen tissue.

$* \rho<0.05$, compared to control for that group.

\section{Age-Related Changes in Brain Monoamines}

A. Carlsson, Department of Pharmacology,

University of Göteborg, S-400 33 Göteborg, Sweden.

In postmortem human brains from patients without any known neurological or psychiatric disturbance the levels of dopamine, noradrenaline and 5-HT have been found to decrease with increasing age. Compared to dopamine, the other two monoamines show a less striking age dependence with significant negative correlation coefficients only in a hemisphere region but not in the brain-stem. Monoamine oxidase activity, measured with $\beta$-phenethylamine as substrate, increased with age. In senile dementia of Alzheimer type dopamine, noradrenaline and 5-HT levels were significantly reduced in the two examined brain regions, i.e. the caudate nucleus and hippoca mpus. Homovanillic acid and 5-hydroxyindoleacetic acid were reduced, though less than the corresponding amines, whereas 3-methoxy-4hydroxyphenyl-glycol was elevated. Choline acetyltransferase activity was reduced and monoamine oxidase activity increased. Chronic alcoholics showed changes similar to senile dementia. It is suggested that Parkinson's disease and senile degeneration, in the former case comprising mainly dopaminergic neurons, whereas in the latter case several types of neurons are involved. However, the demarcation line between the two conditions is not sharp. Chronic alcohol abuse appears to accelerate neuronal aging. The well established age dependence of depressive illness may be explained by the age-related decline of brain monoamines.

\section{Deprenyl in Parkinson's Disease}

M. Casacchia, C. Fieschi, G. Meco and M. Nardini, Dept. Neurol. and Psychiatry, University of Roma, Italy.

Deprenyl is a potent irreversible inhibitor of human platelet MAO,B, which deaminates phenylethylamine, benzylamine and dopamine. This drug was used in the therapy of Parkinson's disease and showed to be effective on the management of L-DOPA on-off phenomena. These patients were treated with deprenyl $10 \mathrm{mg}$. daily during the first two weeks and after with placebo during other two weeks. The results showed that deprenyl had a positive action on on-off phenomena in $30 \%$ of patients, but it produced in many cases side effects, such as, confusion and agitation.

Effect of Brain Phospholipids in Parkinson's Disease. Clinical and Neurological Aspects.

M. Casacchia, R. Cappellini, G. Meco, L. Bove, R. Cirillo-Dept. of Neurol. and Psychiat. University of Rome.

Purified preparation of brain cortex phospholipids (BCPL) are able to elicit biochemical effects in animal and in man, which may be explained in terms of activation of cerebral dopaminergic pathway. BCPL potentiate the stimulation of hypothalamic adenylate cyclase by dopamine. BCPL act less on the activation of striatal or nucleus accumbens adenylate cyclase, because polar molecules cross the blood brain barrier with some difficul ties. The present study is an attempt to investigate a possible effects or BCPL in Parkinson's disease where dopamine funcation is known to be impaired. 20 patients took part in the study. $500 \mathrm{mg}$ of sonicated BCPL suspension where given slowly i.v. Analyzing the results, 13 out of 20 patients showed some improvement on Webster R.S. depressive mood and autonomy. Rigidity and bradikinesia did not change significantly. At the beginning, during and at the end of this study plasma prolactin levels were measured. BCPL alone do not induce any change in prolactin levels. On the contrary BCPL can antagonize the DOPA=IDD increased prolactin: that effect may be interpretated as the result of an effect on dopaminergic system.

Dopamine Agonist Therapy of Naturally Occurring and Drug-Induced Hyperkinetic Extrapyramidal Disorders

T. N. Chase* and C.A. Tamminga, National Institute of Neurological and Communicative Disorders and Stroke, Bethesda, Md. and Department of Psychiatry, University of Chicago, Chicago, II.

In the experimetal animal, dopamine precursors generally induce or potentiate hyperkinetic motor behavior while pharmacologic agents which block dopamine mediated synaptic transmission tend to produce or exacerbate hypokinesia. Similar effects are also observed in patients with naturally occurring or drug-induced extrapyramidal movement disorders such as Parkinson's disease, Huntington's chorea or tardive dyskinesia. Recently, however, increasing evidence suggests that certain dopamine agonists may act to suppress rather than increase some hyperkinetic states. Results of four different dopamine agonists (apomorphine, piribedil, bromocriptine, and CF 25-397) will be compared in patients with tardive dyskinesia and Huntington's chorea with the known pharmacologic actions of these agents. Discussion of these clinical observations will center on possible pharmacologic mechanisms, as well as on implications regarding the management of L-dopa-induced dyskinesias in parkinsonian patients.

A Comparative Study of Dyskinesias and Dystonias Provoked by L-Dopa and Bromocriptine with Special Reference to the "On-Ofr" Effect

C. Chouza, S. Romero, A. Schroeder, J. Lorenzo, M. Gaudiano and J. B. Gomensoro, Institute of Neurology, Montevideo, Uruguay.

Dystonias and dyskinesias were evaluated in 3 groups of patients who were given respectively L-DOPA (39 patients), L-DOPA associated with benserazide ( 37 patients) and bromocriptine ( 31 patients), during 1 year. Dyskinesias were most frequent in the L-DOPA benserazide combination $(78 \%)$, somewhat less frequent with L-DOPA $(69 \%)$ and significantly less frequent with bromocriptine. Dystonias were primarily observed with the combination $(48.6 \%)$, less often with L-DOPA $(10.2 \%)$, and unnoticed with bromocriptine. In a group of 9 patients with "on-off" effect the combination L-DOPA-benserazide was progressively substituted for $70 \mathrm{mg}$ bromocriptine. Direct observation was carried out 14 hours daily at each stage of the test, the parkinsonian symptoms, the dyskinesias and dystonias being evaluated and charted. One patient did not tolerate bromocriptine, 2 worsened with the association and even more with the substitution, 5 improved with the association, with transient disappearance of the "on-off" effect in 3 of them, and in 1 patient there was distinct improvement of the fluctuations and dyskinesias following substitution. Bromocriptine, exhibiting a lower antiparkinsonian effectiveness, resulted in a lower percentage of dyskinesias, did not provoke dystonias and proved to possess a more prolonged therapeutic effect.

\section{Increased Dependence on Visual Information for Arm Movement in} Patients with Parkinson's Disease.

J. D. Cooke and J. D. Brown. Depts. of Physiology and Clinical Neurological Sciences, Univ. Western Ontario, London, Can. Studies by Hore et al. (1) on monkeys revealed an increased dependence on visual information for movement performance during temporary dysfunction of the globus pallidus. We have studied step-tracking movements made with and without visual guidance by patients with Parkinson's disease. The subjects were seated comfortably with their forearm horizontal and supported near the elbow. The subjects grasped a vertical rod attached to a manipulandum handle and were required to move the handle between two target zones using alternating flexion and extension movements about 
the elbow. Target and handle positions could be displayed to the subject on an oscilloscope. Two conditions were tested: a) with both target and handle positions displayed and b) with only target position displayed. During the latter trials it was found that all patients showed a consistent drift of overall arm position towards flexion. Average movement amplitudes were unchanged from trials with visual guidance. The absolute drift in arm position (approximately $20 \mathrm{deg}$ in one minute) was much greater than the reported values for passive displacement threshold in normal humans. Thus although performing appropriate movements in the absence of vision the patients evidenced a lack of appreciation of absolute arm position.

(1) Hore, J., Meyer-Lohmann, J. and Brooks, V. B. Science 195, 584, 1977.

(Supported by PG-1 from the Medical Research Council of Canada).

\section{Functional Organization of the Substantia Nigra, Globus Pallidus and} Subthalamic Nucleus in the Monkey

M. R. DeLong and A.P. Georgopoulos, Dept. Physiology, The Johns Hopkins Sch. Medicine, 725 N. Wolfe St., Baltimore, Md. 21205, U.S.A

Neural activity in the s. nigra pars compacta $(\mathrm{SNpc})$ and pars reticulata (SNpr), the internal (GPi) and external (GPe) segments of the globus pallidus and the subthalamic nucleus (STN) was studied in monkeys in relation to active and "passive" movements of individual body parts as well as during performance on a visuomotor tracking task (Georgopoulos and DeLong, this volume). Neurons in $\mathrm{SNpc}$ had a low discharge rate whereas neurons in $\mathrm{SNpr}$ had a high discharge rate similar to GPi neurons. Units related to arm, leg and orofacial movements were organized in a somatotopic pattern in both GPe and GPi, and STN. The majority of limbrelated units were activated by proximal movements. Only a few limbrelated neurons were found in $\mathrm{SNpr}$, while orofacial units were numerous these were located in the lateral regions of the nucleus. In all the nuclei studied neurons in the more medial and rostral portions were most often unrelated to the task and could not be clearly activated by active or "passive" movements. Cells of the SNpc were not activated by any of the movements or manipulations tried.

The above findings suggest that the SNpr and the GPi together best be viewed functionally as a single output system from the basal ganglia which has been divided by the fibers of the internal capsule. This is suggested by the similarities in the spontaneous discharge rates of SNpr and GPi neurons and by the apparent continuation of the body representation accross $\mathrm{GPi}$ and $\mathrm{SNpr}$, with neurons related to orofacial movements located in the medial parts of GPi and the lateral portions of SNpr. Supporting evidence for this view comes from the known close similarities in the morphology of neurons and neuropil in both structures, and the close parallels in their afferent and efferent connections with the striatum and thalamus. On the other hand, the dopamine continaing neurons of the SNpc appear to constitute a distinctly separate neural system which functions as a tonic system with a more general role in behavior.

\section{Electrophysiological Properties of Identified Output Neurons of the Rat Sustantia Nigra (Pars Compacta and Pars Reticulata): Evidences for the Existence of Branched Neurons \\ J. M. Deniau, C. Hammond, A. Rizk and J. Féger, Laboratoire de Physiologie des Centres Nerveux, Université P. \& M. Curie 75230 - Paris Cedex 05.}

The organization of Substantia Nigra projections to the ipsilateral Striatum, to both VL-VM thalamic nuclei and to both Superior Colliculi was studied in ketamine anesthetized rats. SN output neurons were identified by their antidromic activation after electrical stimulation of these target nuclei. Neurons in the pars compacta were activated by the Striatum only. These cells exhibited a low spontaneous discharges rate ( 3 to 6 per sec.) and their axon had a slow conduction velocity $(0,33$ to $1 \mathrm{~m}$ per sec.). These neurons are presumed to given rise to the dopaminergic nigrostriatal pathway. The pars reticulata neurons are characterized by a higher spontaneous activity ( 20 to $40 \mathrm{per} \mathrm{sec}$ ) and a faster axonal conduction velocity $(1,9$ to $10 \mathrm{~m}$. per sec). These cells were antidromically activated from Striatum, Thalamus and Superior Colliculus. These projections are mainly directed toward the ipsilateral side but some cells could also be activated from the contralateral stimulating sites. An important proportion of these neurons were found to have branching axons projecting at least to two of the dilferent nuclei studied.

J. M. Deniau el al. Exp. Brain Res. 1978, 32.

\section{Comparison of Mortality Rates in Parkinson Patients Treated with Sinemet or Levodopa.}

Shirley G. Diamond and Charles H. Markham, Department of Neurology, UCLA School of Medicine, Los Angeles, California 90024, U.S.A.

Efficacy of levodopa in treatment of parkinson's disease has been well documented. The related question of mortality rate was examined in 1087 levodopa-treated patients, and the ratio of observed to expected deaths was found to be 1.0278 or approximately what is seen in a similar normal population, vastly less than the pre-levodopa mortality of three times normal. Recent studies comparing efficacy of Sinemet and/or Madopa in controlling Parkinson symptoms have generally found inhibitor-containing compounds superior to levodopa alone. This study investigates mortality of patients treated with Sinemet. Twenty-five neurologists across the USA comprised the original group of primary investigators studying Sinemet during its experimental trials in 1972-74, and most have continued to treat these patients to the present. Each was asked for current data on a maximum of 20 patients randomly selected by us. To date 13 investigators have supplied information on 207 patients representing 688 person-years of observation. Expected number of deaths in a group of this composition from the general population is 18.4990 . Observed number in the Sinemet patients was 33, resulting in a ratio of observed to expected deaths of 1.7839. At present we cannot say if the difference in mortality rates of regular levodopa vs. Sinemet lies in the populations studied or in the drugs.

\section{Morphologic and Behavioral Effects of Kainic Acid Injected Into Rat Striatum \\ R. C. Duvoisin and C. Mytilineou,}

Mount Sinai School of Medicine, New York, U.S.A. 10029

The injection of $10 \mathrm{n}$ moles kainic acid into the center of the left striatum of the rat at the level of the anterior commissure produced striking morphologic alterations and behavioral effects. Vigorous contraverse circling and focal motor seizures persisted for 24 to 48 hours following lesioning. Phenytoin abolished the seizures but not the circling behavior. Subsequently, the animals tended to circle spontaneously to the left on handling; this tendency was accentuated by apomorphine and levodopa. Morphologic alteration comprised a severe degeneration of neuronal elements of the neostriatum, pallidium, nucleus accumbens, the amygdaloid nuclei, large portions of the entorhinal cortex, the hippocampus and the lateral reticular nucleus of the thalamus. Fluorescent histochemistry in animals sacrificed 5-7 days after lesioning showed increased fluorescence of dopamine nerve fibres in the striatum, and in the limbic lobe including the cingulate gyrus. This finding is believed to represent inhibition of nigrostriatal and mesolimbic dopaminergic systems due to destruction of the cells of origin of striatonigral GABA projections. A film of the acute behavioral effects will be shown.

Dopamine, Acetylcholine and Estrogens at the Rat Striatum Level C. Euvrard, J.P. Raynaud, F. Labrie and J. R. Boissier C. de Recherches Roussel-Uclaf, 93230 Romainville, France Lab. Endo. Mol., Quebec GIV 4G2, Canada

L-Dopa-induced dyskinesia and tardive dyskinesia following chronic treatment with neuroleptics have been found to be improved by estrogen treatment. Such neurological symptoms have been related to an overstimulation of DA transmission or to a dysfunction of the DA-ACh balance in the extrapyramidal system. As the nigro-striatal DA pathway exerts a direct inhibitory control on the striatal cholinergic neurons, measurements of striatal ACh levels are a good index for the estimation of dopaminergic influences on these neurons. In rats pretreated with the potent estrogen, Moxestrol, the increase in ACh levels induced by DA agonists was considerably reduced; conversely, the decrease in ACh levels observed after acute administration of neuroleptics was markedly potentiated. On the other hand, the effect of harmaline, a potent tremorigenic drug, was also found to be partially blocked by estrogen treatment. More interestingly, Moxestrol led to a significant inhibition of the striatal $\mathrm{ACh}$ increase observed after chronic administration of neuroleptics. As demonstrated by using rats with a unilateral lesion of the substantia nigra, this antidopaminergic action of estrogen is independent of the integrity of the DA nigrostriatal pathway. These results obtained with estrogen at the level of the rate striatum could have important implications in the treatment of Parkinson's disease. 
Electromyographic Responses to Limb Displacement in Parkinson's Disease (PD)

E. V. Evarts, D. E. Beuchert, H.T. Teräväinen, D. B. Calne, Lab. Neurophysiology, NIMH and Experimental Therapeutics Branch, NINCDS, Bethesda, Maryland, U.S.A. 20014

Biceps stretch reflexes were elicited by forearm pronation in PD patients and controls.
DISPLACEMENT
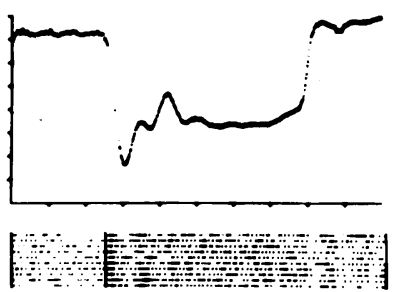

DISPLACEMENT

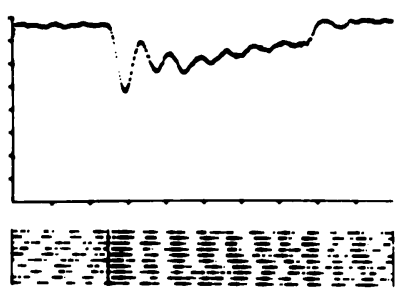

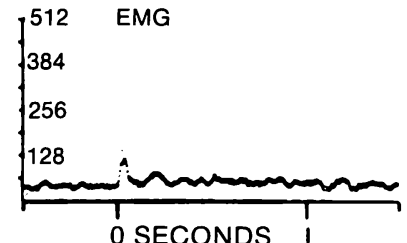

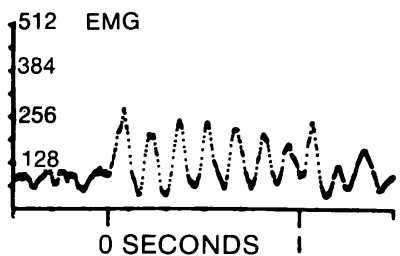

The figure above illustrates displacement and EMG responses to identical torque steps in a rigid PD patient (right) and an elderly control (left). The patient exhibited (1) a higher level of pre-displacement EMG; (2) a smaller displacement in response to the change in torque; (3) a larger EMG reflex response to displacement; (4) resetting and enhancement of tremor. Controls were subdivided according to age (18-24, 25-49, and 50-80 years). Those in the two younger groups had low levels of background EMG activity, but a number of the older normal subjects and, of course, the rigid PD patients exhibited rather high levels of tonic EMG activity even when the prestretch load called for EMG silence. Tremor triggering was confined to PD patients but enhanced amplitude of the $50-100$ ms latency response to stretch occurred both in PD and in normal subjects with increased muscle tone. PD and normal subjects without hypertonus did not show elevated reflex responses.

\section{Increase of Parkinsonian Symptoms as a} Manifestation of Levodopa Toxicity

Stanley Fahn and Robert E. Barrett, Department of Neurology,

Columbia University College of Physicians and Surgeons and the Neurological Institute, New York, N.Y. 10032

Classically, CNS manifestations of levodopa toxicity consist of abnormal involuntary movements, confusion, and psychiatric symptoms of hallucinations and psychosis. The symptoms of start-hesitation and sudden transient freezing are also considered to be manifestations of excess levodopa in some situations. We have observed five patients with parkinsonism who obtained partial or marked imporvement with levodopa therapy and who also demonstrated an increase of parkinsonian symptoms when the dosage was increased. Improvement was seen when the dosage of levodopa was reduced. All five patients were receiving Sinemet, and four of them were on supplemental levodopa. Two patients never had other manifestations of levodopa toxicity, two had postural hypotension, and one had choreic movements. The increased parkinsonian symptoms with levodopa overdosage were primarily an increase of bradykinesia, rigidity, and postural instability with more difficulty walking. Tremor was not encountered.
Peripheral Excitatory Input to Substantia Nigra

J. Féger and C. Ohye, Laboratoire de Physiologie des Centres Nerveux Université P. \& M. Curie 75230 Paris Cedex 05

In chloralose-anesthetized cats, unitary responses were recorded from the Substantia Nigra after electrical and natural stimulation of the extremities. Forty-eight nigral neurons from a total of 67 in the pars compacta as well in the pars reticulata responded to electrical stimulation of either paws or dissected peripheral nerves with a latency of about 15 to 20 ms. Bilateral convergent responses were generally observed with some preferential side for a given neurons. It was revealed that electrical stimulation of the flexor nerve or cutaneous nerve was far more effective in inducing nigral response. It was also demonstrated that passive flexion of the forearm increased the spontaneous discharge whereas passive extension suppressed it. These nigral responses to peripheral stimulation were still recorded after extensive lesion of the diencephalon, thus interrupting all nigral afferent fibers from higher centers. The functionnal role of the flexor afferent projection of the Substantia Nigra is to be discussed. These data can be related with postural characteristics of people with Parkinson's disease:in standing position, there is an hypertony of flexor muscle, providing the typical stooping posture.

J. Féger, J. Jacquemin and C. Ohye Exp. Neurol. 59, 351-360 (1978) Peripheral excitatory input to Substantia Nigra.

The Localization of Acetylcholinesterase in the Corpus Striatum and Substantia Nigra of the Rat

H. C. Fibiger', John Lehmann' and L. L. Butcher'. ' Div. Neurological Sci., Dept. Psychiatry, University of British Columbia, Vancouver, B.C., Canada and ${ }^{2}$ Dept. Psychology, University of California, Los Angeles, California, U.S.A.

The distribution of acetylcholinesterase ( $\mathrm{AChE}$ ) in some extrapyramidal nuclei was examined by means of intrastriatal injections of kainic acid (KA). Pharmacohistochemical experiments showed that AChE-containing neurons in the striatum were among those which were destroyed by kainic acid. In complementary biochemical studies, it was demonstrated that approximately $50 \%$ of the total AChE activity in the striatum was localized in these AChE-containing neurons. Intrastriatal injections of KA produced a substantial decrease in the activity of the glutamic acid decarboxylase in the substantia nigra, thus demonstrating that neurons contributing to the striato-and/or pallidonigral pathways had been lesioned. However, nigral AChe activity was not significantly reduced by the striatal kainic acid injections. Furthermore, stereotaxic injections of colchicine along the course of the striato-nigral projection failed to produce an accumulation of $\mathrm{AChE}$ in these fibers proximal to the injection. In contrast, injections of colchicine into the nigro-striatal projection led to a proximal accumulation of $\mathrm{AChE}$ in the fibers of this system, thus confirming the presence of $\mathrm{AChE}$ in these dopaminergic neurons. It is concluded that the striato-and pallidonigral projections in the rat do not contain AChE. Furthermore, AChEcontaining neurons in the striatum appear to be interneurons rather than the souce of striatal efferents. It is suggested that these AChE-containing neurons may be striatal cholinergic interneurons.

Spontancous Activity of Globus Pallidus Neurons During Alterations of Striatal Dopaminergic Mechanisms in Monkeys

M. Filion, Laboratoires de Neurobiologie, Hôpital de l'Enfant-Jésus et Département de Physiologic, Université Laval, Québec, Canada

The present study tests the hypothesis that lesions of the nigrostriatal dopaminergic pathway result in a functional defect of the striatum that disturbs the activity of pallidal neurons. The spontaneous activity of these neurons was recorded in awake monkeys at rest. A group of animals showed signs of parkinsonism following ventrotegmental midbrain lesions. Recordings were made also in intact monkeys with or without drugs acting on the dopaminergic system. In monkeys with lesions, pallidal neurons display bursting activities continuously, during movement and at rest. This is not the case in intact monkeys not receiving drugs. The number of bursting neurons was recorded in awake monkeys at rest. A group of animals showed degeneration in the pars compacta of the ipsilateral substantia nigra. With completc and bilateral nigrostriatal lesions all medial pallidal neurons 
display bursting activities. This is reproduced in intact monkeys by dopamine antagonists. Dopamine agonists suppress the signs of parkinsonism and the pallidal bursting activities in monkeys with lesions. These results are in accordance with the hypothesis that lesions of the substantia nigra bring a substantial contribution to the development of signs of parkinsonism. (Supported by the MRC)

\section{Studies on Different Types of DA Pathways and Their Interaction} With Glutamate, GABA and Enkephalin Neurons

K. Fuxe, R. Schwarcz, L. Agnati, K. Andersson, I. Hökfelt, M. Pérez de la Mora and L. Ferland. Department of Histology, Karolinska Institute, Stockholm 60, Sweden.

Studies on dotted and diffuse types of DA nerve terminals in tuberculum olfactorium and in nuc. accumbens indicate that the dotted type of DA terminals has a lower amine turnover than the diffuse type of DA terminals. Also the islandic DA terminals in the neostriatum seem to have a lower DA turnover than the diffuse type of DA terminals in neostriatum. An ergolene derivative PTR 17402 can preferentially reduce DA turnover in the neostriatal DA islands. It may be that the DA receptors belonging to the islandic nerve terminals are related to adenylate cyclase (AC), since PTR 17402 can increase $A C$ by $40 \%$. Kainic acid produces after injection into the caudate a marked increase of neostriatal DA turnover. Thus, glutamate receptors may exert an excitatory influence on DA systems in the neostriatum. Met-enkephalin and $\beta$-endorphin can increase DA turnover in some subcortical areas and in parts of the nuc. caudatus following intraventricular injections. Thus enkephalin interneuron can exert excitatory effects on certain types of DA terminal systems of the forebrain. The GABA receptors seem capable of exerting both excitatory and inhibitory influences on DA neurons in forebrain since muscimol increases and GABA transaminase inhibitors decrease DA turnover.

\section{Quantitative Studies of Neuronal Activity} in Basal Ganglia During Limb Movements

A. P. Georgopoulos and M. R. DeLong, Department of Physiology, The Johns Hopkins Sch. Medicine, 725 N. Wolfe Street, Baltimore, Maryland 21205, U.S.A.

The activity of single neurons in the external (GPe) and the internal (GPi) segments of the globus pallidus and the subthalamic nucleus (STN) was studied in the monkey in a step and a pursuit arm tracking task. The experiment was designed to evaluate the effects of flexion and extension movements of different amplitudes (range $12-50 \mathrm{~mm}$, step) and velocities (range $18-31 \mathrm{~mm} / \mathrm{s}$, pursuit) on neurons judged to be related to arm movements by examination of the monkey cutside the tasks. In the step task 168 neurons were studied: 101 in GPe, 38 in GPi and 29 in STN; in the pursuit task, 125: 79 in GPe, 30 in GPi and 16 in STN. We evaluated in a trial-by-trial analysis (a) the changes of the mean discharge rate during the reaction and movement time from a control holding period by a paired $t$ test, (b) the effects of amplitude, velocity and direction of movement by an analysis of variance, and (c) the correlation between changes in mean discharge rate and selected parameters of movement (amplitude, peak velocity, peak acceleration in the step task).

It was found that (1) many neurons were activated in all 3 structures with both tasks, both during the reaction and movement time: STN was the most "movement-related" structure of the three (all STN neurons studied showed significant changes during movement time, M.T., and $62 \%$ during the reaction time, R.T., step data), with GPi second ( $84 \%$ in MT and $63 \%$ in RT) and GPe last ( $74 \%$ in $\mathrm{Mt}$ and $56 \%$ in RT), (2) increase of the activity over the control period occurred more frequently than decrease, (3) directional effects were prominent, the proportions of cells more active with flexion or extension being very similar in each structure and for both tasks, (4) cells preferentially related to amplitude (small vs. large) or the velocity (fast vs. slow) of the movement were infrequent and, when they occurred, they were nearly equally distributed among the above classes, and (5) changes in mean discharge rate were uncorrelated with parameters of movement in the large majority of neurons.
Autoradiographic Study of the Projections of the Basal Ganglia, Substantia Nigra and Red Nucleus in the Cat and Monkey

M. Giguère, R. Goucher and L. J. Poirier, Laboratoires de neurobiologie, Hôpital de l'Enfant-Jésus et Département d'Anatomie, Université Laval, Qué. Canada.

In the cat nigral efferents originating in the two medial thirds of the substantia nigra give rise to two distinct bundles which are better visualized at rostral midbrain level. These two bundles predominantly end in the ipsilateral pallidum and in both divisions (compacta and reticulata) of the homolateral substantia nigra. Very few striatal efferents, if any, terminate in the entopenducular nucleus. Entopenducular efferents terminate massively in the lateral nucleus of the habenula and the centrum medianum on the corresponding side. In addition entopenducular efferents reach other structures of the contralateral side through the supramamillary commissure. In the monkey the nigral efferents were also observed to end in the striatum according to a topographical arrangement. In this species the striatal efferents are also distributed topographically to the pallidum and to both divisions of the substantia nigra on the corresponding side. In both species the rubrotegmentospinal tract projects massively to the contralateral lateral reticular nucleus and the rubro-olivary fibers reach certain areas of the ipsilateral inferior olive. (Supported by the Medical Research Council of Canada).

The Influence of B-Adrenergic Blocking Agents on Parkinson Syndrome

F. Gerstenbrand, J. Rainer and W. Poewe,

University Clinic/Neurology Innsbruck, A-6020

During the past 10 years different authors reported remarkable good effects on the essential tremor due to $\beta$-blocking agents. Few and contradictional reports have been published about the $\beta$-blockers influence on rigidity and akinesia. Because of the insufficient efficacy of L-Dopa on Parkinsonian tremor, the aim of our first study was to proof the influence of $\beta$-blocking agents on Parkinsons disease. We have treated 10 patients $(4$ females, 6 males, average age 62, suffering from Parkinson's disease, dominated by tremor, showing mild- 4 , moderate- 4 , and moderate severe- 2 form within a 5 graduate scale) with Bupranolol in a double blind study, which lasted 4 weeks. The dose was $100 \mathrm{mg}$ for 1 week and $200 \mathrm{mg}$ for the 2nd week respectively 1 to 2 placebo. For the evaluation we used doctors-, selfrating-, GRUENBERGER-, BERGER-, BRICKENKAMP-, $100 \mathrm{~mm}$ -writing-drawing-test and HAMILTON scale. All the patients had abstable L-dopa treatment. We found out, that the tremor mainly decreased but also rigidity akinesia and emotional condition have been improved. We also treated a second group of more 10 patients in an apen study with Bupranolol in a dosage of $150 \mathrm{mg}$ and Propranolol 80 to $120 \mathrm{mg}$ with similar effects as in the double blind study. 5 patients suffering from posttraumatic Parkinson signes combined with cerebellar symptoms in consequence of a traumatic apallic syndrome we treated with $\beta$-blockers, too. Increased metabolism, Parkinson's symptoms as well as the cerebellar symptoms have been markedly improved. Summarizing we could see that $\beta$-blockers combined with L-dopa were able to ameliorate the global parkinson's symptomatology and even some cerebellar symptoms.

\section{Presynaptic Control of Dopamine Release in the Striatum}

M.F.Giorguieff, M. L. Kemel, J.Glowinski, M.J. Besson, Groupe NB, Collège de France, Paris.

In order to investigate the intrastriatal regulation of the release of dopamine (DA) from dopaminergic nerve terminals, the effect of some neurotransmitters normally present in striatal nerve endings was studied in vitro. Rat striatal slices were superfused with an artificial CSF containing $\mathrm{L}-3,5-{ }^{3} \mathrm{H}$-trosine $(5 \mathrm{Ci} / \mathrm{m} \mathrm{M}, 50 \mathrm{uCi} / \mathrm{ml})$ and the newly synthesized ${ }^{3} \mathrm{H}-\mathrm{DA}$ spontaneously released was measured in serial superfusate fractions. When added to the superfusion medium, acetylcholine $(\mathrm{ACh})\left(10^{-6} \mathrm{M}, 10^{-5} \mathrm{M}\right)$, GABA $\left(10^{-5}\right.$ to $\left.10^{-3} \mathrm{M}\right)$ and L-glutamic acid $\left(5.10^{-5} \mathrm{M}\right)$ enhanced ${ }^{3} \mathrm{H}-\mathrm{DA}$ release through an action on pharmacologically different receptors. The $\mathrm{ACh}$ induced release of ${ }^{3} \mathrm{H}-\mathrm{DA}$ is reduced in the presence of muscarinic or nicotinic antagonists. The stimulatory effect of $\mathrm{ACh}$ and of L-glutamic acid persist in the presence of tetrodotoxine, suggesting that cholinergic and glutamatergic neurons could directly modulate the striatal DA release through a presynaptic mechanism. In opposite, the effect of $\mathrm{GABA}$ on ${ }^{3} \mathrm{H}$ DA release completely disappeared in the presence of tetrodotoxine. 
The Antiparkinsonian Efficacy of Ergot Alkaloids

M. Goldstein, J. Y. Lew, S. Nakamura, A. F. Battista, A. Lieberman, Departments of Psychiatry, Neurosurgery and Neurology, Neurochemistry Laboratories, New York, New York 10016

The antiparkinsonian efficacy of several alkaloids and their interactions with putative dopamine receptors were investigated. Bromocriptine and lergotrile relive the surgically induced tremor in monkeys and produce less severe abnormal involuntary movements than L-dopa. Serveral hitherto not reported ergots were found to be more potent antitremor agents than bromocriptine or lergotrile in experimental monkeys. Clinical studies indicate that bromocriptine and lergotrile are therapeutically effective in parkinsonian patients. Ergot alkaloids compete effectively for the binding of radio-labeled dopamine agonists as well as for dopamine antagonists to striatal membrane sites. Lergotrile has a higher affinity for ${ }^{3} \mathrm{H}$-dopamine and for ${ }^{3} \mathrm{H}$-apomorphine binding sites than bromocriptine. Bromocriptine is more potent in competing for the binding of ${ }^{3} \mathrm{H}$-haloperidol or ${ }^{3} \mathrm{H}$ spiroperidol than lergotrile. The high affinity of bromocriptine for displacing of ${ }^{3} \mathrm{H}$-spiroperidol or ${ }^{3} \mathrm{H}$-haloperidol suggests that this ergot may interact with a population of dopamine receptors that functionally differ from other populations of dopamine receptors. Acute and chronic treatment with bromocriptine elicits, up to 6 hours after termination of the treatment, a decrease in the binding of ${ }^{3} \mathrm{H}$-spiroperidol. This is probably due to the accumulation of the drug in the striatal membrane. Chronic treatment with a potent dopaminergic ergot (14I-B) elicits a decrease in the binding of ${ }^{3} \mathrm{H}$-spiroperidol to striatal membranes. Thus treatment with potent dopamine agonists may produce a tolerance to the drug. (Supported by grant NS06801-12)

\section{Dyskinesia and the "On-Off" Phenomenon}

A-K Granérus, Department of Geriatric and Long Term Care Medicine, Vasa Hospital, University of Göteborg, Göteborg, Sweden

In December 1975 a follow up was performed in eighty-five parkinsonian patients treated with L-dopa, alone or combined with a peripheral dopa decarboxylase inhibitor, for five years or more. "On-off" symptoms had appeared in 43 of these 85 long term treated patients, apparently with a greater risk of such symptoms to occur the younger the patient had been at the beginning of the disease and at the start of the L-dopa treatment. The Ldopa dose had been higher throughout the treatment, the initial improvement had been more marked and dyskinesia had occurred earlier during the treatment in the patients later developing "on-off" symptoms than in those maintaining an even effect. In 91 per cent of the patients with later occurring "on-off" symptoms, compared to 40 per cent in the patients without such symptoms, the first appearance of dyskinesia was seen within the first year of L-dopa treatment. At the time of the follow up all the patients in the "on-off" group had dyskinesia, more or less often, which at least partly was due to the symptomatology connected to the "on"-periods. The occurrence of dyskinesia, thus, seemed to imply a warning for an "onoff" phenomenon to develop later, perhaps especially if there was a marked initial improvement.

Choline and Lecithin Administration to patients with Tardive Dyskinesia J. H. Growdion, New England Medical Center Hosptial, Boston, MA 02111

Choline $(\mathrm{Ch})$ is the physiologic precursor of acetylcholine ( $\mathrm{ACh}$ ); its availability in brain influences the rate at which neurons synthesize ACh. Lecithin (Lec) is the naturally occurring dietary source of $\mathrm{Ch}$; either $\mathrm{Ch}$ or Lec may be given to treat diseases, such as tardive dyskinesia (TD), by enhancing cholinergic tone. Thirteen patients with facial and limb dyskinetic movements took oral doses of Ch or Lec: 10 had an associated history of neuroleptics (TD), and 3 did not. Clinical response was rated by visual movements counts, AIM's scores and review of movies. Seven of 10 patients with TD took $10-16 \mathrm{~g}$ per day of $\mathrm{Ch}$ for $>1$ month. During this time, mean plasma $\mathrm{Ch}$ levels rose $154 \%(\mathrm{p}<.001) 1$ hour after ingestion; movements decreased in 5, did not change in 1 and increased in 1 . Three of the patients who improved with $\mathrm{Ch}$ also improved during ingestion of an equimolar dose of Lec and 2 of 3 patients who did not take Ch also improved; mean plasma $\mathrm{Ch}$ levels rose $159 \%(\mathrm{p}<.001)$. Ch did not alter the number of movements in the 3 patients with idiopathic dyskinesia.

\section{Electrophysiological Demonstration of an Excitatory Subthalamo-Nigral Pathway in the Rat with Special References to Branched Subthalamonigral Output Neurons}

C. Hammond, J. M. Deniau, A. Rizk and J. Féger, Laboratoire de Physiologie des Centres Nerveux Université P. \& M. Curie, 75230 Paris Cedex 05

The subthalamo-nigral projection was studied with extracellular techniques in the rat. Orthodromic and antidromic stimulation of this pathway was performed in different sets of experiments in order to exclude the effects due to the possible activation of passing fibers. The majority of the recorded cells in the Subthalamic nucleus (STN) were antidromically activated by nigral stimulation. Furthermore it was shown that the STN output neurons are excitatory to cells located in the pars compacta as well in the pars reticulata of the Substantia Nigra (SN). It is noteworthy that this excitatory response was still recorded in the $\mathrm{SN}$ of animals with a chronic low hemitransection (plane A 6.5). In the homolateral $\mathrm{SN}$ and pedunculus of these animals, an important fiber degeneration was observed (degeneration of striato-nigral axons as well as other descending afferents to the $\mathrm{SN}$ ). The major STN efferent system already known is the subthalamo-pallidal pathway. Coupled stimulation of Globus Pallidus or Entopeduncular nucleus and SN showed that some STN cells could be antidromically activated from two $(32 / 81)$ or three $(15 / 81)$ of these structures. Collision tests were performed in order to demonstrate clearly the axonal branching of these STN output neurons.

\section{Projection of the Entopeduncular Nucleus to the Habenula in the Cat.} An Anatomical and Physiological Study

C. Harnois, M. Filion and A. Parent, Laboratoires de Neurobiologie, Hôpital de l'Enfant-Jésus et Départments d'Anatomie et de Physiologie, Université Laval, Québec, Canada

Anatomical studies suggest that the entopeduncular (EN) nucleus of the cat sends a substantial projection to the habenula. Acordingly, during a study of this projection, we observed that neurons of the EN nucleus can be excited antidromically by electrical stimulation of the habenula and that they can be labelled retrogradely by injections of HRP in the habenula. Moreover, some EN neurons are excited antidromically not only by stimulation of the habenula but also by stimulation of one, of two, or even of three other projection sites of the EN nucleus: the ventrollateral and the centromedian thalamic nuclei, and the pedunculopontine midbrain nucleus. However, only $25 \%$ of the total population $(\mathrm{N}=237)$ of En neurons excited antidromically respond to the habenula, whereas 68,69 and $54 \%$ respond to the ventrolateral, to the centromedian and to the pedunculopontine nuclei, respectively. Moreover, stimulation of the habenula excites antidromically more neurons located in the lateral hypothalamus and in the preoptic area than in the nearby EN nucleus. Injections of HRP in the habenula give similar results. Thus, some En neuron project to the habenula, a structure of the limbic system, but more EN neurons project to structures of the extrapyramidal system and not to the habenula. (Supported by the MRC).

\section{Electronmicroscopic Differentiation of the Extrinsic and Intrinsic Types of Synapses in the Striatum and their Putative Transmitters}

R. Hassler, Max-Planck-Institut für Hirnforschung, Frankfurt a/M, FRG.

After having distinguished nine types of synapses in each of the three main parts of the striatum - putamen, caudate and fundus striati (nucleus accumbens septi) - a vertically oriented tissue column measuring $5 \mathrm{~mm}$ in diameter was isolated from all afferent and efferent connections in order to determine which striatal neurons and which types of synapses are intrinsic or extrinsic. More than $90 \%$ of all perikarya belonging to the category of small "spiny" neurons which usually lack any axo-somatic contacts, remain intact and show only cytoplasmic swelling. The perikarya and stemdendrites of "aspiny" giant or large neurons that so bear axo-somatic synapses, undergo a dark or penumbral degeneration. The extrinsic axo-spinous synapses type I (dopaminergic, arising from the nigra), type Ill (glutaminergic, arising from the cortical areas), and type IV (cholinergic, arising from the centre median), as well, undergo dark degeneration of the boutons within the tissue cylinder. This irreversible antegrade dark degeneration is seen, too, in tha axo-somatic (or axo-dendritic) boutons en passant type II (dopaminergic, arising from the nigra), in type VI (of unkown origin), and 


\section{Every leading}

pharmaceutical house
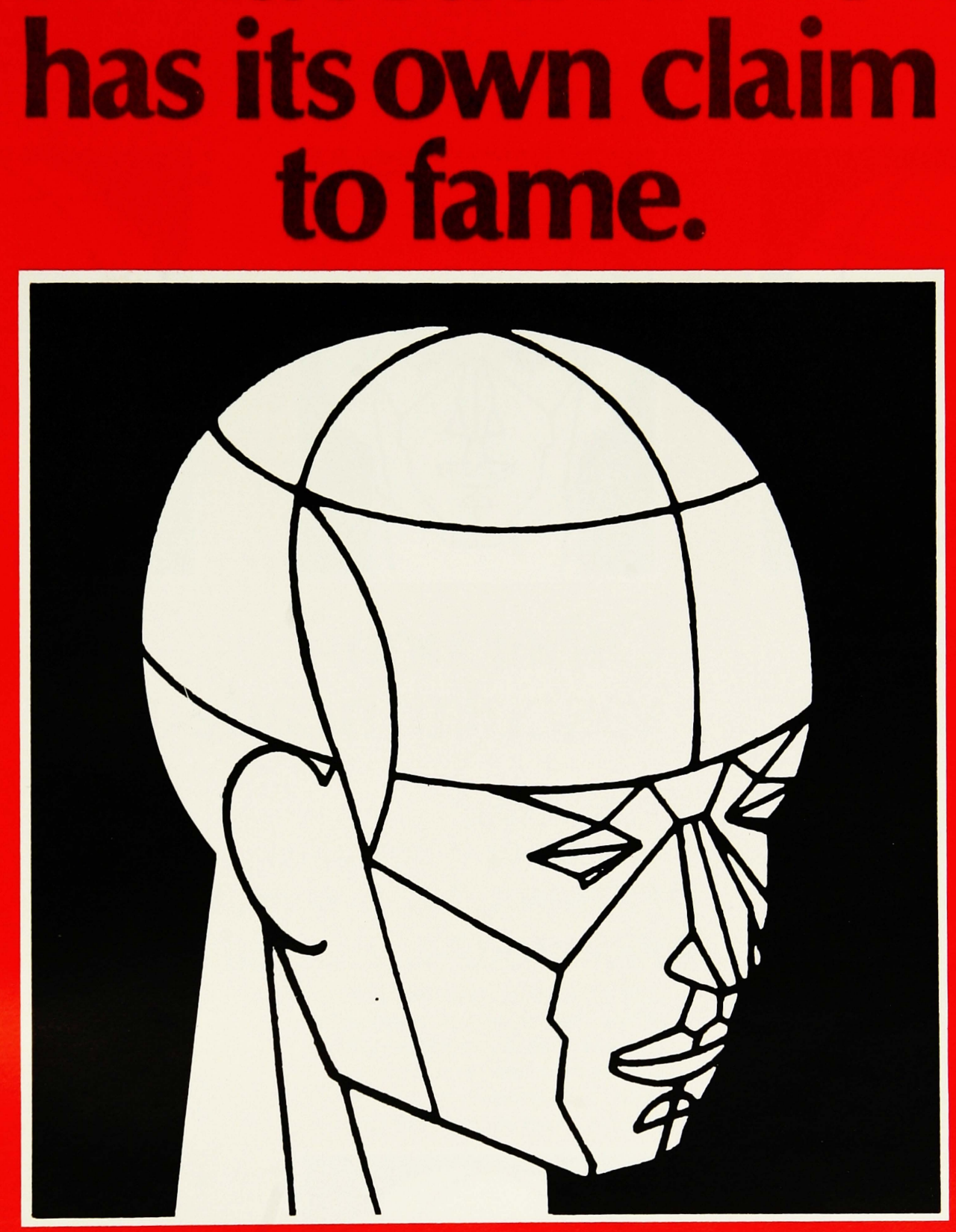

Ours is headache therapy.

\section{SANDOZ}

The leader in headache research and treatment. 
Vascular headaches

of the migraine type

ICAFERGOT ${ }^{\circledR}$ tablets PGYNERGEN ${ }^{\circledR}$ tablets

and injections

Symptomatic treatment of

classic, common, or cluster

migraine.

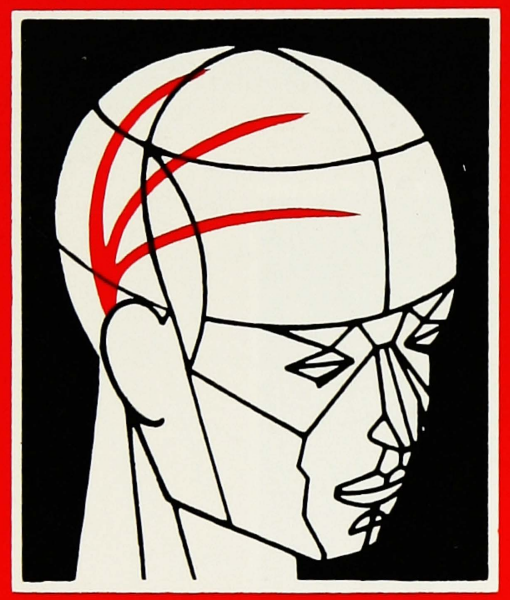

Tension

headaches

(muscle contraction)

\section{FIORINAL ${ }^{\circledR}$}

tablets and capsules

(1) FIORINAL-C $1 / 4$

capsules

(1)FIORINAL-C $1 / 2$

\section{capsules}

\section{Symptomatic treatment of}

muscle contraction headache

(tension headache)

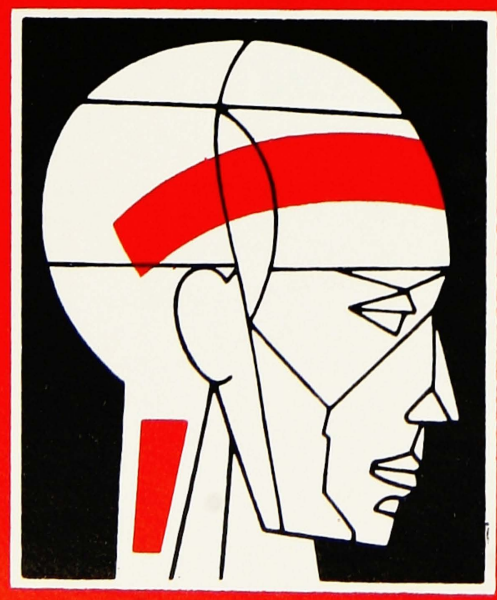

ISANDOMIGRAN ${ }^{\circledR}$ tablets

ISSANSERT ${ }^{\circledR}$ tablets

Prophylactic treatment of

frequent, recurring vascular

headaches

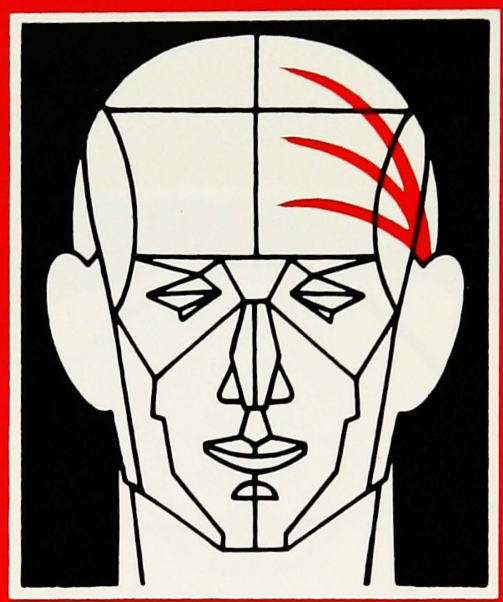

$\bullet$ CAFERGOT`-PB tablets and

\section{suppositories}

\section{Symptomatic treatment of} classic, common, or cluster migraine (accompanied by nervous tension, nausea and vomiting)

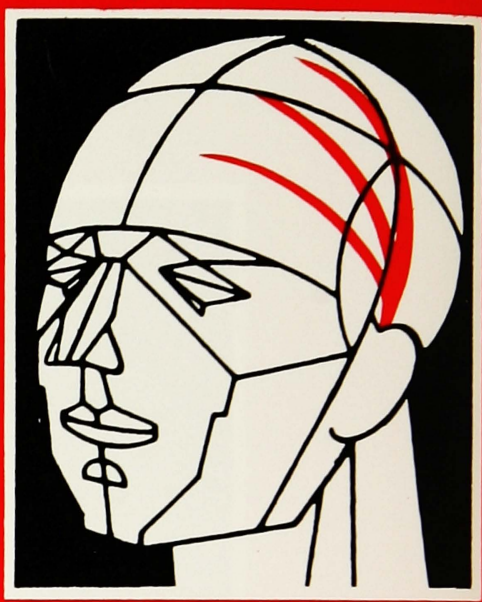

Other non-

vascular headaches

\section{FIORINAL ${ }^{8}$}

tablets and capsules

(1)FIORINAL-C $1 / 4$ capsules

Q FIORINAL ${ }^{\circledR}-\mathrm{C} 1 / 2$ capsules

Symptomatic treatment of

other non-vascular headaches

(headaches associated with

dysmenorrhea, sinusitis,

febrile diseases, cold and

grippe, overeating, hangover).

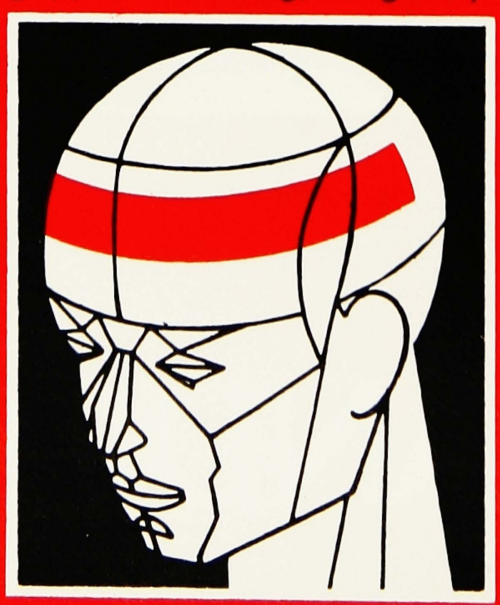

Full product information is available upon request

Contact your Sandoz representative or write to the Medical Services Department of Sandoz (Canada) Limited for a complimentary supply of our new diagnostic aid - the patient's "HEADACHE HISTORY' or for information about our audio visuals concerning the diagnosis and treatment of headaches.

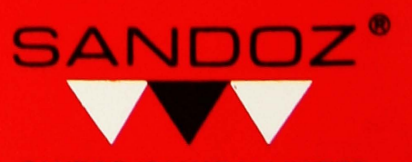


in type VII (either glutaminergic - arising from the cortical areas - or cholinergic, arising from the centre median). The axo-somatic synapses with large round vesicles (type IX) remain almost always undamaged, although a few of them show a swollen bouton. They are the main type of intrinsic synapse, and seem to arise from the small "spiny" neurons which then connect to the "aspiny" neurons using acetylcholine as a transmitter. The other axo-somatic type $V$ with pleomorphic GABA-ergic vesicles, which are almost fully preserved, are, in fact, intrastriatal, GABA-ergic axon terminals of the strio-nigral fibers. In the fundus striati, the type VIII dendritic terminals with small, round vesicles suspended between neurofilaments in an almost clear dendroplasm, usually do not undergo degeneration. However, in the caudate nucleus the same type of synapses frequently show swollen and deformed vesicles. They are presumed to originate from the dwarf cells which are also preserved in our experiments.

Monkey Arm Movement Performance During Basal Ganglia Dysfunction J. Hore and T. Vilis, Physiology Department, University of Western Ontario, London, Canada N6A 5Cl

The performance of 8 Cebus monkeys, which made prompt elbow flexion and extension movements in response to a visual signal, was examined during reversible cooling in the ventral-lateral region of the putamen and globus pallidus. Cooling in this region produced severe disorders in both flexion and extension movements which included: rebound of the limb towards its initial position after onset of movement, slowed movements, and occasionally, delayed onset of movement. These disorders were observed in monkeys making either fast or slow movements. Once the disorder occurred, monkeys showed great reluctance in continuing to make movements. Analysis of EMG activity revealed that these movement disorders were primarily a result of earlier onset and increased magnitude of antagonist muscle activity. No delay was observed in the earliest onset of agonist activity, although an increase in tonic agonist activity usually occurred when holding in target. Perturbations applied to the handle revealed no major changes in segmental or suprasegmental stretch reflexes during cooling.

These results indicate that the basal ganglia do not contribute to the initial phasic agonist muscle activity which results in movement onset. In keeping with recent discoveries about the basic processes underlying arm and head movements (1) it is suggested that the basal ganglia participate in movements by specifying the equilibrium point between agonist and antagonist muscles for the end position of the movement. Failure to correctly change the equilibrium point at the start of a new movement during basal ganglia dysfunction, would result in the observed disorders.

Experiments are currently being performed in which motor cortex discharge is recorded during basal gagnlia dysfunction.

1. Bizzi, E. and Polit, A. Neuropsychologia. In Press. (Supported by the Canadian MRC, PG-1)

\section{Compensatory Neurotransmitter Changes in the Parkinsonian Brain: Effect of Dopaminergic Drug Treatment}

Oleh Hornykiewicz, Inst. Biochem. Pharmacol., Univ. of Vienna, Austria, \& Clarke Inst. Psychiat., Toronto, Canada.

The dopamine (DA) deficiency in the striatum in Parkinson's disease has two functional consequences which have to be considered as compensatory in nature; they seem to subserve the purpose of maintaining a critical minimum of function for as long periods of time as possible in a disease which basically is both degenerative and progressive. These two functional changes are: (a) overactivity of the remaining striatal DA neurons; and (b) supersensitivity of the striatal DA receptors. Effective L-dopa therapy of Parkinson's disease has been shown to increase the levels of the striatal DA. This $\mathrm{Da}$ accumulation can be expected to reduce, via the known negative fee dback loop, the activity of the remaining DA neurons, thus eliminating one important compensatory change. Likewise, the striatal DA receptors may become desensitized by the accumulating DA. There is evidence to show that the latter event in fact takes place in the striatum of patients on longterm L-dopa therapy. The desensitization of the striatal DA receptors may represent an important factor limiting the success of longterm L-dopa therapy. This may help to explain the clinical observation that with time the patients' response to L-dopa definitely declines. Perhaps intermittent Ldopa therapy would better preserve the responsivity of Parkinsonian patients to dopaminergic drug therapy.
Role of Cerebral Cortex in Tremor Mechanisms Reevaluated in Light of Ach-Biogenic Amine Interactions on Cortical Cells

H. Jasper, T. Reader and J.de Champlain, Centre de recherche en sciences neurologiques, Département de physiologie,

Université de Montréal, Montréal, Québec, Canada. H3C 3T8

Attention to dopamine and $\mathrm{ACh}$-dopamine, and GABA interactions in nigro-striatal interactions has caused a neglect of the possible importance of cerebral cortex in tremor mechanisms as suggested by the original observations of Parkinson himself. The importance of motor cortex in the control of experimental tremor in the Poirier midbrain preparation has been demonstrated in previous studies.

Studies of the rate of release of $\mathrm{ACh}$, dopamine and noradrenaline from cerebral cortex in the cat has shown important interactions between $\mathrm{ACh}$ and catecholamine release probably acting in part at the presynaptic level. Iontophoretic studies with multibarrelled micropipettes has shown reciprocal interactions between ACh and DA or NA on cortical cells, NA and DA causing a long lasting inhibition of spontaneous and $\mathrm{ACh}$ induced discharge of cortical neurones. These results, in addition to other evidence from studies of ACh, DA and NA interactions in the peripheral autonomic system suggest that the catecholamines may be important regulators or modulators of activity in cortical cells, and consequently of possible importance in our understanding of the effect of $\mathrm{ACh}$ and catecholamines upon Parkinsonian-like tremor. (Supported by the MRC Canada)

Striato-Nigral Relationship: Morphological and Electrophysiological Studies

S. T. Kitai, J. D. Kocsis, R. J. Preston and C. P. VanderMaelen, Dept. of Anatomy, Michigan State University, E. Lansing, MI U.S.A. 48824

Caudate (Cd) neurons in cats anesthetized with $\alpha$-chloralose were analyzed for responses to electrical stimulation of the cerebral cortex $(\mathbf{C x})$, the centromedian-parafascicular (CMP) area of the thalamus, the substantia nigra (SN) and the internal capsule (IC). Intracellular recordings were obtained through microelectrodes filled with $4 \%$ horseradish peroxidase (HRP) in $0.5 \mathrm{M} \mathrm{KCl-tris} \mathrm{buffer.} \mathrm{Stimulation} \mathrm{of} \mathrm{CMP,} \mathrm{SN} \mathrm{or} \mathrm{Cx} \mathrm{elicited}$ mon'osynaptic EPSPs. The EPSPs were often followed by hyperpolarizing potential lasting $100 \mathrm{msec}$ or more. In some neurons, stimulation of $\mathrm{SN}$ or IC evoked constant latency spikes that were not triggered by EPSPs. The antidromic nature of these spikes was determined by collision of antidromic spikes with intracellularly induced orthodromic spikes. These cells were marked by electrophoresing HRP through the intracellularly positioned recording electrode. The HRP filled neurons were then reconstructed serially with the use of a microscope and a drawing tube. Neurons responding antidromically were found to have the somadendritic morphology of the spiny neuron. In conclusion, $\mathrm{Cd}$ projection neurons are spiny neurons and these neurons receive convergent monosynaptic excitatory inputs from the thalamus, substantia nigra and the cerebral cortex. (Supported'by NIH Grant NS 00405.)

Effect of Chronic Levodopa Treatment on Striatal Membrane Dopamine Binding

H.L. Klawans, A. Hitri, P.A.Nausieda and W.J. Weiner, Rush Medical College, Chicago, Illinois 60612.

The relationship between duration of levodopa therapy and the incidence and severity of levodopa induced dyskinesias raises the possibility that chronic dopamine agonism may play a role in the pathogenesis of these abnormal involuntary movements. We have been using stereotyped behavior in rodents as a model of such dyskinesias as both are felt to reflect dopamine activity at striatal dopamine receptors and have previously reported that chronic levodopa administration to guinea pigs produces increased behavioral response to apomorphine. This suggests that such chronic therapy induces dopamine receptor hypersensitivity. In order to test this hypothesis we have studies $\mathrm{H}^{3} \mathrm{DA}$ binding to striatal membranes of guinea pigs pretreated for three weeks with levodopa in a dose which has been demonstrated to produce behavioral supersensitivity. Two distinct sites were observed in control animals. At the high affinity binding site chronic levodopa treatment produced a 4 fold increase of affinity without affecting the number of binding sites. At the low affinity binding sites chronic levodopa increased the number of binding sites without modifying 
the affinity. This data provides direct evidence that chronic levodopa treatment produces alterations in dopamine receptor sensitivity which may be important in the pathophysiology of levodopa induced dyskinesias.

\section{The Anterior Pituitary Dopamine Receptor: A Model for CNS Dopaminergic Systems}

Fernand Labrie, Michèle Beaulieu, Thérèse Di Paolo, Louise Ferland and Vincent Raymond, MRC Group in Molecular Endocrinology, Le Centre Hospitalier de l'Université Laval, Québec, G1V 4G2, Canada.

The specificity of binding of $\left[{ }^{3} \mathrm{H}\right]$ dihydroergocryptine, $\left[{ }^{3} \mathrm{H}\right]$ sprioperidol, $\left[{ }^{3} \mathrm{H}\right]$ haloperidol, $\left[{ }^{3} \mathrm{H}\right]$ dopamine, $\left[{ }^{3} \mathrm{H}\right]$ apomorphine and $\left[{ }^{3} \mathrm{H}\right] \mathrm{R} \mathrm{U} 24213(\mathrm{~N}$ propyl $\mathrm{N}$-phenethyl m-tyramine) to bovine adenohypophyseal plasma membranes is typically dopaminergic and shows a close correlation with the control of prolactin release in rat anterior pituitary cells in primary culture studied with a large series of dopamine (DA) agonists and antagonists. The high degree of precision of the pituitary cell culture system can be used to measure the DA agonistic and/or antagonistic activities of compounds of potential use in Parkinson's disease, schizophrenia and hyperprolactinemia. A close correlation could be found between changes of prolactin release in pituitary cells in culture and the pharmacological and clinical potencies of a large series of DA agonists and antagonists. The pituitary system is free of the limitations of the DA receptor binding and adenylyl cyclase assays previously used as screening assays. Estrogens have been found to exert a potent antidopaminergic activity at the pituitary level, this action being exerted at a step following binding of DA agonists to the receptor. Similar effects of estrogens are exerted at the level of the striatum.

\section{Neuropharmacological Investigation and Treatment of Spasmodic Torticollis}

S. Lal, K. Hoyte, M. E. Kiely, T. L. Sourkes, D. W. Baxter and F. Anderman, McGill University, Montreal, Quebec, Canada. H3G 1Y6

The effect of a single dose of various drugs was assessed in 13 patients with spasmodic torticollis, mainly by placebo-controlled videotape recording and blind evaluation. The number of subjects with significant improvement $(\mathrm{n})$ was as follows:

\section{Drug}

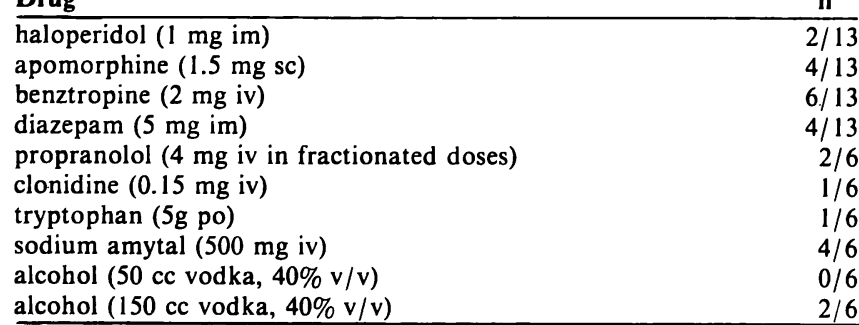

Daily treatment with benztropine (2-8 $\mathrm{mg}$ /day), pimozide $(4-6 \mathrm{mg} /$ day) or L-dopa (2.0-3.0 g/day) improved $4 / 9,2 / 8$ and $2 / 6$ patients, respectively. All 4 patients with a sustained therapeutic effect with benztropine, but none of the 5 therapeutic failures, had improved following a single iv dose of the drug. Only 2 of the 4 patients who responded to apomorphine improved with L-dopa. Methysergide $(8 \mathrm{mg} /$ day) was ineffective in 4 cases.

Spasmodic torticollis is a heterogeneous disorder. Response to a single dose of benztropine has predictive value in selection of pharmacotherapy.

\section{Reflex Mechanisms in Parkinsonian Tremor and Essential Tremor}

R. G. Lee, and R. B. Stein, Department of Medicine (Neurology), University of Calgary and Department of Physiology, University of Alberta

Tremor may originate as a result of abnormal oscillations in peripheral reflex loops or it may be driven by central oscillators which develop rhythmical activity independent of sensory input. To investigate the degree to which reflex mechanisms contribute to the generation of human pathological tremors, we applied randomly timed mechanical perturbations at the wrist and examined the extent to which these stimuli were capable of resetting the rhythm of the tremor. The deviation between expected time and actual time of occurrence of EMG bursts was calculated for a number of tremor cycles following the stimuli, and these values were plotted as a function of the time within the tremor cycle where the stimulus occurred. The slope of a straight line fitted to these points provided a Resetting Index $(R I)(0=$ no resetting; $1=$ complete resetting). Patients with essential tremor all showed a high degree of resetting - the mean value for the RI for 5 patients was 0.63 . Parkinsonian patients appear to fall into two groups; 4 patients showed almost no resetting with RI's less than 0.2 while 4 other patients had RI's within the same range as the values for essential tremor. Reflex mechanisms therefore appear to play an important role in essential tremor. The variable results in Parkinsonism may relate to the relative involvement of the nigrostriatal and olivocerebello-rubral systems.

\section{Measurement of Tetrahydrobiopterin in Human Cerebrospinal Fluid \\ R. A. Levine, D. Robinson, A.C. Williams, M. H. Ebert, W. Lovenberg, NHLBI, NIMH, NINCDS; National Institutes of Health, Bethesda, MD. U.S.A. 20014}

Recent studies have indicated a regulatory role of tetrahydrobiopterin (THB) as cofactor for aromatic amino acid hydroxylases in the synthesis of catecholamines and serotonin. Measurement of THB in human CSF might serve as an index for central aminergic activity. To obtain the necessary sensitivity for THB measurement in human CSF, a modification of the system described by Guroff et al. for measuring hydroxylase cofactor activity in tissue was developed. This modified radioenzymatic assay utilized highly purified rat liver phenylalanine hydroxylase. The ${ }^{3} \mathrm{H}$-tyrosine formed from ${ }^{3} \mathrm{H}$-phenylalanine was reacted with iodosuccinamide to release tritium as ${ }^{3} \mathrm{HOH}$. Our studies indicated this hydroxylation system was linear with respect to incubation time up to 45 minutes. The reaction rate was proportional to THB added between 1 and 160 pmol. This assay appears specific for reduced biopterin since purfied dihydrofolate added to this system did not enhance hydroxylation activity. Lyophilization of CSF aliquots permitted measurement of THB as low as $2 \mathrm{pmol} / \mathrm{ml}$. The mean THB level in CSF of normal subjects was $17.7 \mathrm{pmol} / \mathrm{ml}$. There was a positive correlation $(r=.73)$ between THB and HVA but not between THB and 5HIAA in CSF. It is concluded that measurement of TH B in CSF may provide a useful index of central dopaminergic function.

\section{Therapeutical Approach of L-Dopa Induced Onset and End of Doses Dyskinesias}

F. Lhermitte, J. L. Signoret, A. M. Bonnet, Y. Agid, Clinique de Neurologie et Neuropsychologie, Hôpital de la Salpêtrière, Paris, France

Abnormal movements provoked by L-DOPA were observed in 99 out of 165 patients with Parkinson's disease and were divided in two groups. 77 patients experienced "mid-dose dyskinesias" occuring at the maximum therapeutic effect. 22 patients exhibited "onset and end of dose" dyskinesias (mid-dose dyskinesias were present in 13 of these patients) characterized by four essential features: 1) the nature of the underlying Parkinsonism, i.e. young age at onset of disease, severity of akinesia and quality of response to L-DOPA; 2) the ballic and dystonic features of the involuntary movements producing an extreme disability; 3) occurrence of dyskinesias at the beginning and end of the period of effectiveness of a dose of L-DOPA, coinciding with rise and fall in plasma levels of DOPA; 4) aggravation of dyskinesias by dopamine blocking agents, and reduction of dyskinesias by an increase and fractionation of the daily dose of L-DOPA

Bromocriptine and Lergotrile: Comparative Efficacy in Parkinson Disease A. N. Lieberman, M. Kupersmith, I. Casson, R. Durso, S. H. Foo,

M. Khayali, T. Tartaro, New York University, School of Medicine, Dept. of Neurology, New York, U.S.A.

Bromocriptine and lergotrile were administered at different times to 81 patients with advanced Parkinson disease (PD) and increasing disability despite optimal treatment with levodopa. Sixty-six patients were treated with bromocriptine of whom 45 tolerated at least $25 \mathrm{mg}$./day (adequately treated); and 53 patients were treated with lergotrile of whom 39 tolerated at least $20 \mathrm{mg}$./day (adequately treated). Both adequately treated groups had significantly decreased rigidity, tremor, brady-kinesia, gait disturbance, total score, and stage upon addition of bromocriptine or lergotrile to levodopa. Twenty-five patients improved at least one stage on bromocriptine, and 21 improved at least one stage on lergotrile. Nineteen of 27 patients with "on-off" effects improved on bromocriptine, while 13 of 21 patients with "on-off" effects improved on lergotrile. The mean dose of bromocriptine in adequately treated patients was $47 \mathrm{mg}$., and the mean dose of lergotrile was $49 \mathrm{mg}$., permitting a $10 \%$ reduction in levodopa. Bromocriptine was discontinued in 29 of 66 patients because of adverse effects, including mental changes (14 patients) and involuntary movements 
(9 patients). Lergotrile was discontinued in 33 of 53 patients because of adverse effects including hepatotoxicity (11 patients), mental changes (12 patients), and orthostatic hypotension (8 patients). While the results of trẹatment with bromocriptine or lergotrile were comparable, there were differences among individual patients with some improving more on bromocriptine and others more on lergotrile. Both drugs were useful in the management of patients with advanced disease, and suggest that even more effective agents may be developed.

\section{GABA and 3H-GABA Binding in Parkinson's Disease and Huntington's Chorea}

K. G. Lloyd, L. Shemen and L. Davidson, Synthélabo-LERS, 31, ave P. V. Couturier 92220 BAGNEUX, France and

Clarke Institute of Psychiatry, Toronto, Canada

The functional aspect of GABA neurons in neurological disease is a topic of current clinical concern. New non-toxic GABA-related drugs are becoming available which potentially can be used in GABA deficiency syndromes. In Parkinson's disease glutamic acid decarboxylase (GAD) activity is decreased in the substantia nigra and striatal nuclei and $3 \mathrm{H}$ GABA receptor binding is decreased in the substantia nigra but not the striatum. These observations suggest that GABA receptors exist on dopamine (DA) nigral cell bodies or dendrites but that relatively few (in relation to the total striatal GABA receptors population) occur on the striatal DA terminals. The decrease in GAD is interpreted as a sign of a compensatory decrease of an inhibitory input to DA neurons, allowing these neurons to fire at a higher rate. Therefore inspite of the apparent GABA deficiency in Parkinson's disease treatment of this condition with GABA mimetic drugs would seem unwise. Huntington's chorea also shows evidence of a GABA deficiency. In contrast to Parkinson's disease, $3 \mathrm{H}-$ GABA receptor binding is severely decreased in the caudate and putamen. The remaining receptors may have an increased affinity for GABA indicating a rationale for GABA mimetic therapy. In cerebellar membranes from Huntington's patients, the affinity for $3 \mathrm{H}-\mathrm{GABA}$ binding is greatly increased $(\mathrm{Kd}=43 \mathrm{nM}$ as compared to $116 \mathrm{nM}$ for controls). This alteration is reproduced by treating material from control brains with Triton-X-100 or phospholipase-C posibly indicating that: (a) an alteration in membrane phospholipids is responsible for the changes seen in $3 \mathrm{H}-\mathrm{GABA}$ binding; and (b) the accessability to, or the affinity of, the GABA receptor is normally controlled by membrane phospholipids.

\section{Cytohistochemical Study of the Primate Basal Ganglia and Substantia Nigra}

R. Marchand, A. Parent and L.J. Poirier, Laboratoires de neurobiologie, Hôpital de l'Enfant-Jésus and Départment d'Anatomie, Université Laval, Québec, Qué., Canada

On the basis of Nissl material and of the distribution of acetylcholinesterase (AChE) five types of neurons may be identified in the substantia nigra of the monkey. Types $\mathrm{Cl}$ and $\mathrm{C} 2$ are present in the pars compacta. Type $\mathrm{Cl}$ neurons which have medium-sized and ovoid cell bodies most likely correspond to the dopaminergic neurons. The fewer type $\mathrm{C} 2$ neurons have triangular or pyramidal cell bodies and processes which show an important $\mathrm{AChE}$ activity. Type $\mathrm{L}$ neurons mainly located in the lateral and ventrolateral parts of the substantia nigra have rounded or triangular cell bodies. The Nissl substance has a punctiform distribution. They are very similar to those of the pallidum. Type $\mathrm{R} I$ neurons which are intensively stained in Nissl material occupy the ventromedial area of the substantia nigra. Type R2 neurons with small cell bodies are diffusely distributed. In AChE material the perikarya and processes of two types of neurons of the neostriatum, representing a small percentage of all neurons in this structure, are intensely stained. In addition numerous large and multipolar neurons associated with the lenticular nucleus (intralamellar and peripallidal groups) including nucleus ansae lenticularis show an intense AChE activity. (Supported by the Medical Research Council of Canada).

\section{Abnormal Behavior and Myoclonic Seizures in Rats Following} Stereotaxic Neostriatal Injection of Herpes Simplex Virus

Charles H. Markham and II Jin Bak, Department of Neurology, UCLA School of Medicine, Los Angeles, California 90024, U.S.A.

Three to four days after herpes simplex virus (HSV) is injected into one striatum, certain neurons were selectively infected. These included relatively few striatal neurons and many neurons in the ipsilateral substantia nigra, dorsal raphe and in the cerebral cortex bilaterally. At the same time, the rats showed characteristic behavioral abnormalities. They showed wetness about the eyes, nose and mouth. Circling, when it took place, was toward either the ipsilateral or contralateral side. Myoclonic seizure activity was manifested by repeated flexor movements of the ipsilateral toes, feet or forelimbs, sometimes occurring every second or two and sometimes as a brief flurry of three or four movements in one second. Myoclonic movements occurred on both sides of the face and there were marked repetitive extension movements of the head and neck.

Quite different behavior followed injection into the temporal lobe or frontal cortex.

A motion picture will be shown illustrating this behavior.

\section{The Effects of Altered Motor Control on Motor Units}

R.F. Mayer, Department of Neurology, University of Maryland School of Medicine, Baltimore, Md., USA 21201 and Laboratory of Neural Control, NINCDS, NIH, Bethesda, Md. USA 20014

This report will summarize some of the data which we have obtained in experimental animals and man concerning montoneuron and muscle unit properties following motor dysfunction. In the cat, long-term immobilization of ankle extensors and flexors at $90^{\circ}$ in one hind limb with steel pins, results in muscle atrophy (medial gastrocnemius, MG) which is greatest in FR fibers and least in FF fibers (Burke, Kanda and Mayer, 1975). There is no change in the percentage of motor unit types in the MG but there is significant $(p<0.001)$ decrease in mean twitch and tetanic tensions in each motor unit type especially in the $\mathrm{FR}$ and $\mathrm{S}$ units. There also is a significant $(p<0.001)$ decrease in the mean amplitude of composite homonymous (MG) and heteronymous (LG S) Ia EPSPs in each unit type (Mayer, Burke, Kanda, Walmsley and Hodgson, 1978). Preliminary studies in cats with long-term paraplegia following spinal cord transection (T12-13) have shown a significant $(p<0.01)$ decrease in mean twitch and tetanic tensions in each unit type but no change in MG or LG S Ia EPSPs. This data suggests that long-term limb immobilization with an intact nervous system can alter synaptic strength possibly by a decrease in spindle afferent activity. In rats rendered paraplegic (14-270 days) by intraspinal 6aminonicotinamide the decrease in mean twitch and tetanic tensions is greater in soleus than extensor digitorum longus (EDL) and is associated with a decrease in mean area of all fiber types, especially type IIA and IIC fibers (Mano, Mano, Deshpande and Mayer, 1977). In the EDL, there is an increase in the number of type I fibers, usually in small groups, suggestive of increased peripheral sprouting of type I motor units. In man with acute hemiplegia, preliminary studies of the mechanical responses of single muscle units in the first dorsal interosseus muscle suggest that mean twitch tensions are similar to controls but contraction times prolonged. Results will be discussed in relation to motor function.

\section{Bromocriptine Versus Amantadine. A Double Blind Study G. Meco and M.Casacchia, Dept. of Neurol. and Psychiat. University of Rome}

The long-term L-DOPA treatment produces, after many years, several side-effects, as on-off phenomena; actually L-DOPA causes some disturbances of other monoamine transmitters in the nervous system and may contribute to the above mentioned side-effects. On the contrary we can hypothesize that an optimal benefit should be attainable by a selective dopamine agonist. This drug has many advantages over L-DOPA, at least from a theoretical point of view. Bromocriptine is a potent dopaminergic agonist, that seemed to have an antiparkinsonian action in previous studies. We have investigated the effects of bromocriptine vs amantadine in a double blind study, with a sample of 20 untreated parkinsonian patients. We have also studied the action of this drug in association with LDOPA+ID. This research found that bromocriptine was an effective antiparkinsonian drug. It was more effective than a mantadine. It also had a potentiating action on L-DOPA+ID in low doses. This drug decreased the on-off phenomena in some patients. 
Striatal Evoked Excitation and Inhibition

in the Globus Pallidus and Substantia Nigra

J. J. Miller and D. P. Rutherford, Dept. of Physiology,

University of British Columbia, Vancouver, Canada V6T IW5

The effects of stimulation of the striatum on unit activity in the globus pallidus (GP) and regions of the substantia nigra were investigated in urethane anaesthetized rats. Two contrasting response patterns were recorded from GP neurones. For a particular recording site, widely distributed regions of the striatum elicited a positive-going slow potential and inhibition (100-250 msec) of spontaneously firing neurones. This response was then followed by a rhythmical discharge pattern for periods up to $1 \mathrm{sec}$. Relatively confined loci elicited a sharp negative deflection superimposed on the positive-going waveform and an initial spike activation coincidental with the latency of the negative field response $(4 \mathrm{msec})$. This excitation was followed by a period of inhibition $(100-280 \mathrm{msec})$ and the characteristic rhythmical discharge pattern.

Neurones in the substantia nigra pars compacta were antidromically evoked by stimulation of the striatum $(2.8-4.1 \mathrm{msec})$ and nigrostriatal bundle (1.2-3.2 $\mathrm{msec})$. Spontaneously firing cells responding in this manner characteristically exhibited a short $30-50 \mathrm{msec}$ period of inhibition following the antidromic response. Neurones of the pars reticulata antidromically identified by VL thalamic stimulation, exhibited an activation $(5-8 \mathrm{msec})$ or activation-inhibition sequence following single pulse stimulation of anterior and ventral regions of the striatum. Stimulation sites in the posterior striatum and GP produced inhibition of both compacta and reticulata neurones. These data provide evidence for a topographical organization of the efferent excitatory and inhibitory projections of the striatum to pallidal and nigral target neurones. (Supported by the MRC of Canada.)

\section{Nicotinic Receptor Mediated Cholinergic Excitation in the Rat Neostriatum}

U. Misgeld, Max-Planck-Institut f. Hirnforschung, Neurobiologische Abtlg., Frankfurt/M, Fed. Rep. of Germany

The transmitter and receptor generating the locally evoked excitatory potentials were studied in slices of rat neostriatum. Intracellularly, EPSPs and orthodromic or antidromic action potentials were recorded following intrastriatal stimulation. The antidromically and synaptically triggered spikes were seen in extracellular unitary recordings as discharges of short and long latencies, respectively, and in field potential recordings as two consecutive negative waves. $90 \%$ of 240 neurones recorded extracellularly responded to the stimulation with synaptically triggered discharges; most of them could be activated antidromically in addition. Synapses of neighbouring neostriatal neurones were found in deafferentation experiments to be the source of this synaptic excitation. The transmitter of these intrinsic excitatory synapses is acetylcholine, since the synaptic excitation is blocked by anticholinergic drugs and enhanced and prolonged by cholinesterase inhibitors. The excitation was susceptible to dihydro- $\beta$-erythroidine, mecamylamine and hexamethonium, but not to atropine and $\alpha$ bungarotoxin. It was also affected by the synthetic anticholinergic drug biperiden. Nicotinic receptors on neostriatal neurones could therefore play a role in the anticholinergic treatment of extrapyramidal motor disturbances.

\section{“On-Off" Response Studies}

F. H. McDowell and P. Papavasiliou, Dept. of Neurology, Cornell Unviersity, Medical College, New York, 10021, U.S.A.

We have attempted to improve our understanding of the history of Parkinson's Disease by focussing on those patients who have maintained a rather stable existence through ten years of treatment with Levodopa, Levodopa with a decarboxylase inhibitor or Levodopa with other dopamine agonists added to the treatment program. The number of such patients is quite small but they have several things in common. In general, they began with mild to moderate Parkinson's Disease, two, they have continued to lead active, productive lives with enormous support from family members. In recent studies of a response to Levodopa, these individuals clearly probably absorb and metabolize L-Dopa in a different way than do those individuals with rapid fluctuation and response to the medication. Among patients with a steady response, blood levels of dopa following ingestion rise to a relatively low level and are maintained at that level throughout the day. Those with an unstable response have widely fluctuating serum dopa levels and clinical responses. The implications of these differences between patients who have had a steady, consistent, good response to treatment as compared to these who have had a good response but an unpredictable one to treatment, will be discussed.

\section{Ten Year Follow-Up of One Hundred Patients with Parkinsonism Treated with Levodopa}

F. H. McDowell and R. Sweet, Dept. of Neurology, University, Medical College, New York, 10021, U.S.A.

Ten years ago, we began a treatment program for patients with Parkinson's Disease with Levodopa. During the ensuing ten years, the programs of administration of medication were changed but all ultimately rested firmly on the use of Levodopa with or without decarboxylase inhibitors. In the ten years following our admission of our first patient, we have followed the original hundred patients treated in our group in effort to give some evidence of the natural history of treated Parkinson's Disease, its outcome and complications. To date, almost 50 percent of the original one hundred patients have died. The interesting thing about the causes of death is that fewer than usual of the patients have succumbed to complications of atherosclerotic vascular disease or cancer. Among the survivors, over half of the patients show some definite evidence of loss of intellectual capacity or dementia which interferes with the conduct of their daily lives. Another small proportion, as many as 20 percent are beginning to show some impairment in intellectual function which is disturbing their ability to continue a normal active existence. Approximately two-thirds of the surviving patients show disturbing problems with the "on-off" effect with reversion to Parkinsonian symptoms for parts of the day, back to near normal function for unpredictable parts of the day. An additional disturbing complication has been that of postural instability. Falling, start hesitation, festinating gait and freezing are common accompaniments of the surviving individuals in our treatment group. To date, none of these complications have been amenable to any treatment programs we have attempted, including the use of other dopamine agonists or treatment programs. What is striking, is that a very small number of patients throughout the ten year period have maintained a stable response to Levodopa, have not shown remarkable progression of the disorder and continue to maintain an active existence. This group, while small, is important and deserves the special studies which we are now undertaking.

\section{Biochemical pathology of Huntington's Disease and the Kainic Acid "Model"}

E. G. McGeer, P. L. McGeer, T. Hattori, and S. R. Vincent, Kinsmen Lab., UBC, Van., Canada V6T IW5

Injections of kainic acid (KA) into the neostriatum of rats produce lesions similar in many respects to those in Huntington's disease (HD). Morphologically, there is a loss of neurons in the neostriatum. Biochemically, there are decreases in GABAergic and cholinergic indices, in dopaminergic receptors, in encephalin, in substance $P$, in angiotensin converting enzyme and in KA, glutamate and colchicine binding. There are, however, normal (or elevated) levels of dopaminergic indices, of serotonin and of glutamate uptake. The "model" differs from HD in lack of genetic aspects and time course of neuronal death but may provide clues as to the mode of cell death in HD. Evidence will be reviewed supporting the hypothesis that KA neurotoxicity involves action of KA at an extrajunctional receptor which sensitizes the postsynaptic neuron to gluta mate released from afferent neurons; the glutamate action may also be prolonged because KA inhibits uptake. A key experiment is the near abolition of KA's neurotoxicity by prior lesioning of the corticostriatal glutamate tract. The possibility that cell death in HD is related to this tract is an important field for investigation. (Supported by grants from the Huntinton's Disease Foundation, the W. Garfield Weston Foundation and the Medical Research Council of Canada).

\section{Dendroaxonic Communication in the Extrapyramidal System}

\section{P. L. McGeer, Kinsmen Lab., UBC, Vancouver, Canada V6T I W5}

Neurotransmitter communication across axo-dendritic synapses has always been considered to be one-way, i.e. axon to dendrite. Dendritic localization of various neuro-transmitters and synthetic enzymes (tyrosine hydroxylase $(\mathrm{TH})$, tryptophan hydroxylase, choline acetyltransferase (CAT) and glutamate decarboxylase (GAD) has been demonstrated, as has dendritic release of dopamine. Electron microscopic evidence will be 
presented that the TH (and 5-hydroxydopamine) and CAT in dendrites of the substantia nigra (SN) and neostriatum (NCP), respectively, are associated with smooth endoplasmic reticulum, microtubules and vesicular-like structures which are often close to the postsynaptic membrane. Measurements of spiroperidol binding in the SN and of QNB and $\alpha$-bungarotoxin binding in the NCP in rats with lesions designed to produce selective alterations in pre- and post-synaptic processes indicate that muscarinic receptor sites in the NCP are on neuronal perikarya or dendrites but nicotinic receptor sites are on afferent dopaminergic and corticostriatal neurons; in the SN spiroperidol binding is on afferent striatonigral neurons. Both areas lack axo-axonic synapses. The hypothesis is advanced that communication at many CNS synapses involves a neurotransmitter dialog with dendritic release of material to act upon axonal endings. If such dialogs exist, they have important implications for the mechanism and therapy of extrapyramidal disorders.

Caudate Nucleus Activity in Relation to Voluntary Movement J. T. Murphy, J. W. Aldridge and R. J. Anderson, Dept of Physiology, University of Toronto, Toronto, Ontario, Canada, M5S 1 A8

Recordings from single neurons were made in the head of the contralateral caudate nucleus in awake primates during a performance of visually and somatically guided hand movements. Populations of cells showed temporal correlation of discharge patterns with visual or somatic disturbances or both. The mean latency for the responses of 62 caudate neurons to visual disturbances was $203 \mathrm{msec}$, while that for 68 cells to somatic disturbances was $180 \mathrm{msec}$. Analyses of these discharges with respect to the compensating movement triggered by the disturbances showed that these discharges occurred in general about or after the onset of this movement. These findings indicate that the observed cells did not participate in the initiation of the compensating movements, and may instead have acted as part of an internal feedback loop whose function is unrelated to movement onset. (Supported by Medical Research Council of Canada).

\section{Catecholamine Enzymes in Parkinson's Disease}

T. Nagatsu, T. Kato, I. Nagatsu, R. lizuka and H. Narabayashi, Dept. Life Chem., Tokyo Inst. Technol., Yokohama; Dept. Anat., Sch. Med., Fujita-Gakuen Uinv., Toyoake, Aichi; Dept. Psychiatry, Dept. Neurology, Juntendo Univ., Tokyo (Japan)

The activities of tyrosine hydroxylase (TH), dopa decarboxylase (DDC) dopamine- $\boldsymbol{\beta}$-hydroxylase (DBH), phenylethanolamine- $\mathrm{N}$-methyltransferase (PNMT), monoamine oxidase (MAO) A (serotonin as substrate), MAO B (phenylethylamine as substrate), and dopamine (DA)-stimulated adenylate cyclase (AC) were measured in catecholaminergic regions of human brain from 10 controls and 5 patients with Parkinson's disease who had not taken L-dopa during the last 6 months before death. TH activity was markedly decreased in substantia migra, caudate nucleus, putamen and pallidum in all five cases of Parkinson's disease. DDC activity in these regions was also decreased in 3 patients, but two cases had only decreased TH and normal DDC activities. DBH activity in locus coeruleus and hypothalamus and PNMT activity in hypothalamus were also reduced in Parkinsonian cases. Basal AC activity was found to be present in homogenates of caudate nucleus both in controls and in Parkinsonian patients. AC activity was slightly stimulated by DA at $10-100 \mu \mathrm{M}$, and the stimulation was completely blocked by $100 \mu \mathrm{M}$ chlorpromazine. DAstimulated AC activity was significantly higher in Parkinsonian patients than in controls.

\section{Dose and Time Dependency of the Pattern of L-DOPA Induced Dyskinesias in Normal Monkeys and Monkeys with Ventromedial Tegmental Lesions \\ S. Nakatani and A. K. Ommaya, Department of Neurosurgery, Osaka University Medical School, Osaka, Japan and \\ Surgical Neurology Branch, NINCDS, NIH, Bethesda, MD, USA}

Experimental production of L-dopa induced dyskinesias has been reported in rats, cats and monkeys by short term administration of massive doses of the drug. However, it is apparent that such short term effects of $L$ dopa are different from abnormal involuntary movements which emerges in Parkinsonian patients on higher doses but not on massive doses and generally appears after long term administration of L-dopa. Introduction of unilateral lesions into the ventromedial tegmental areas in monkeys induces model syndrome of extrapyramidal dysfunction and a similar biochemical deficiency state of the brain proposed for the Parkinsonian patients. To normal and to such model monkeys, L-dopa/carbidopa combination was administered consecutively up to three months to compare the long term effects with that of short term administration. Ldopa/carbidopa induced distinctively different pattern of dyskinesias in two groups of monkeys at acute and chronic stages. The $16 \mathrm{~mm}$ movie will be shown to demonstrate this remarkable difference. The dreadful ultimate features of L-doa induced dyskinesias will especially be depicted. As the pattern of abnormal movements is not only dose-dependent but also timedependent, a great caution must be excersized in interpreting the results of pharmacological experiments in such model animals carried out attempting to prevent the development of dyskinesias by some chemical agents theoretically supposed to be effective. By the same reason, we feel that either denervation or chronic agonist hypersensitivity of dopaminergic receptors in the striatum alone does not explain the pathogenesis of this movement disorder. It might not be caused merely by a direct effect of dopamine on its receptors. It seems more reasonable to assume that it is based on the background secondary biochemical changes induced by chronic administration of L-dopa, including neurotransmitters other than dopamine. We believe that the monkeys with ventromedial tegmental lesions are the very best biochemical model of Parkinsonian patients in human.

\section{Disturbances of Rhythm Formation in Parkinsonism}

R. Nakamura, H. Nagasaki, R. Taniguchi and H. Narabayashi, Tokyo Metropolitan Institute for Neurosciences, Fuchu-city, Tokyo, Japan. 183

Festination or freezing phenomenon of parkinsonism was observed in the finger tapping test. Some patients could not maintain a synchronous response to the periodic sound signal at a critical frequency around $2.5 \mathrm{~Hz}$ and showed a hastened tapping of 5-6 Hz independent of signal frequencies (Hastening Phenomenon: HP). Due to HP the patients could not make rhythmic motor performances, e.g., gait, correctly. When the signal was cut off in the course of responsive tapping, HP was released at a lower frequency than $2.5 \mathrm{~Hz}$, thus the input signal prevented the release of HP to some extent. The patients with HP was not perfectly suppressed by L-dopa or thalamotomy. In particular, parkinsonian patients were encountered with a specific difficulty of the rhythm formation at $2.5 \mathrm{~Hz}$. Improvement in rhythmic motor performances could be expected, if $\mathrm{HP}$ at $2.5 \mathrm{~Hz}$ were overcome.

\section{Analysis of Two Factors, Psychological Stress Effect and Motor Effect, Influencing on the Involuntary Movement H. Narabayashi and T. Chida, Juntendo Unviersity Hospitals, Tokyo, Japan}

The intentional movement in the severely handicapped patient is considered to necessitate the tense psychological stress to perform the ordered delicate task exactly. The authors applied very simple maneuver to separate two components, i.e. influence by slight motor loading by asking the supine resting patient to hold the arm at elbow-flexed position with $90^{\circ}$, and influence by psychological tension by asking the supine resting patient to make simple calculation or counting back of the numerical numbers.

Huntington's chorea, hemiballism, parkinsonian tremor, and Dopa induced dyskinesia are not influenced or increased by motor loading but worsened markedly by psychological loading.

Postural or intention tremor and posticteric athetoid are increased by motor loading and are further worsened by additional psychological loading.

The first group is due to the diencephalic pathophysiology and the latter group is due to diencephalic and cerebellar pathophysiology. These findings may suggest the nature of the deficit of cerebello-cerebral impluses and the physiological role of cerebellum to manifestation of the diencephalically produced involuntary movement.

\section{Rigidity and Tremor of Neck Muscles in Parkinsonism \\ H. Narabayashi, N. Yasui and Ch. Ohye, Juntendo University Hospitals, Tokyo, Japan}

Sidedness of tremor in neck muscles (sternocleidomastoid muscle, SCM and splenius capitus muscle, $\mathrm{Spc}$.) have been analysed in relation to the sidedness of extremity symptom in 40 cases of parkinsonism and several other conditions, such as dystonic type of cerebral palsy, dystonia and postural or cerebellar tremor. 
Rigidity of neck muscle is somewhat intricate. It appears almost always contralateral to the extremity-side, and homolateral to the side of brain pathology. Effect of thalamotomy on rigidity is contralateral to extremities but ipsilateral to SCM. Spc. is innervated mostly bilaterally but more intensive innervation is contralateral.

Tremor in SCM and Spc. is observed on the same side of extremitytemor, i.e. contralateral to the affected side of the brain.

Since the severity and phenotype of tremor has been known as modifiable depending on the grade of co-existing rigidity as reported in Zürich Symposium (1972) by Narabayashi et al., several combination of these two symptoms on bilateral neck muscles can be drawn.

Existence of such discrepancy in sidedness between rigidity and tremor of neck muscles is important, because it may suggest that the different anatomical structure and physiological mechanism in the brain might be responsible in production of each of these two symptoms.

\section{Role of Dopamine in the Substantia Nigra in the Regulation of the Activity of the Nigro-Striatal Dopaminergic Neurons \\ A. Nieoullon, A. Chéramy and J.Glowinski, Collège de France, 11, place Marcelin Berthelot, 75231 PARIS CEDEX 5.}

The first evidence for a functional role of dopamine (DA) in the substantia nigra (SN) was obtained from electrophysiological studies which showed that the microiontophoretic application of DA reduced the activity of the dopaminergic neurons. Using the push-pull cannula method and the continuous labelling of the dopaminergic nerve endings with ${ }^{3} \mathrm{H}$-tyrosine, the release of ${ }^{3} \mathrm{H}-\mathrm{DA}$ was measured in the cat caudate nucleus during the application of exogenous DA in the corresponding SN using a second pushpull cannula. Results showed that the release of ${ }^{3} \mathrm{H}-\mathrm{DA}$ in the caudate nucleus was reduced by the application of DA at the level of the dopaminergic cell bodies and also by nigral application of amphetamine and benztropine. On the contrary, haloperidol and thioproperazine stimulated the release of ${ }^{3} \mathrm{H}-\mathrm{DA}$ in the striatum when introduced into the superfusing fluid of the push-pull cannula inserted in the SN. These results support the concept of an autoregulation of the activity of the dopaminergic neurons by Da released in the $\mathrm{SN}$.

\section{Glutamic Acid as Possible Neurotransmitter of Neo-And Allocortical Projections to the Basal Ganglia}

C. Nitsch, R. Hassler, J. K. Kim and K.S. Paik, Department of Neurobiology, Max-Planck-Institut für Hirnforschung, D-6000 Frankfurt/M.-71, G.F.R.

Kim et al., Divac et al. and McGeer et al. demonstrated independently in 1977 a significant decrease in glutamic acid (GLU) content and GLU uptake, resp., in rat striatum after lesions of the frontal motor cortex, thus suggesting that GLU might be the transmitter of this pathway. The fundus striati (Nc. accumbens septi) was not studied by these authors. In contrast to the caudate-putamen, the fundus receives in addition to the cortical projection a dense termination of hippocampal fibers. Recently, we (Nitsch et al.) were able to demonstrate substantial decreases in GLU content in the efferent projection fields of the hippocampus, after extirpation of this structure. Therefore, we got interested to compare alterations in GLU content in fundus and caudate after lesions of these neo- and allocortical areas.

In rats, there was performed either a large ablation of the frontal cortex, or a complete uni- or bilateral hippocampusextirpation by sucking out this structure through a small hole in the parietal cortex. Rats with lesions of the parietal cortex served as sham-operated controls. The animals were allowed to survive for 3 weeks, after which regional brain areas were dissected out and GLU concentration estimated using an enzymaticfluorometric assay.

In the hippocampectomized rats, there was found, besides substantial decreases in GLU content in septum, corpus mammillare and contralateral hippocampus, also a significant GLU decrease in fundus striati. In caudate, GLU remained unchanged after hippocampusextirpation, but decreased substantially after frontal cortex lesion. Ultrastructural investigations revealed degenerating boutons in fundus striati after allocortical lesion. These will be compared with the degenerations found in this region after frontal cortex ablation. The results are suggesting that the fundus receives GLUergic input both, from hippocampus and frontal cortex, whereas the GLUergic afferents to the caudate are only of neocortical origin.
Constant Relief (In Man) and Constant Production (In Monkey) of Tremor. A Pathophysiological Study.

C. Ohye, S. Imai, H. Nakajima, T. Shibazaki and T. Hirai, Dept. of Neurosurgery, Gunma Univ. Maebashi, Gunma, 371-Japan

From recent experiences of stereotactic thala motomy on various kinds of tremor, it is established that thala mic ventralis intermedius (Vim) nucleus is an ideal target for the constant relief of tremor. The following criteria of Vim have been proposed: 1) neural noise is significantly high, 2) tremor time locked rhythmic discharges are often found, 3) neurons may respond to passive or active movement of the contralateral extremity but not to light touch. Without exception, this is the first sensory neuron encountered in anteroposterior approach, 4) electrical stimulation of this thalamic point produces paresthesia around its peripheral receptive field at threshold intensity and the movement accompanied by EMG grouping discharge at suprathreshold intensity. A small selective lesion including this thalamic point results in the arrest of tremor without noticable neurological deficit.

By refined stereotactic lesion in the mesencephalic ventromedical tegmental area, parkinsonian like tremor was constantly produced in monkey. Stereotactic recordings were made from cerebellum, brain stem and upper cervical cord by means of microelectrode, and several tremor time locked discharges suggesting descending rhythmic activities were analysed.

Comparative Study of the Intralaminar Thalamic Nuclei Including the Centre Median

An Histochemical Study in the Rat, Cat and Monkey. A. Oliver and A. Parent, Dept. of Neurology \& Neuro-Surgery, Montreal Neurological Institute and Laboratoire de neurobiologie, Laval University.

Montreal, Qué. H3A 2B4 and Quebec City, Qué. G1J 5B3

With the help of the Koelle and Karnovsky-Roots thiocholine technique for the demonstration of acetylcholinesterase and using material from animals pre-treated with DFP, a comparative topographic and cellular analysis was carried out of the intralaminar nuclei, including the centre médian, in the Rat, the Cat and Monkey. The preferential distribution of acetylcholinesterase within the lamellar nuclei of all species and the similarity of their cellular morphology has rendered their homologies easier to recognize. Furthermore, this approach has clarified the sub-division of the thala mic nuclei by stressing the natural boundaries created by the highly reactive lamellar nuclei. In this way, it is easier to determine the basic plan upon which the unclear configuration of the thalamus is built up. Emphasis has been placed on thalamic nuclei closely linked with the striatal system, such as the parafascicular - centre médian complex, the centralis medialis and the paracentralis nuclei. The peculiar morphology of these nuclei in the Cat will be stressed.

Movement Related Brain Macropotentials During Skilled Performance in Parkinson's Disease

D. Papakostopoulos and N. K. Banerji, Burden Neurological Institute, Bristol, and Musgrove Park Hospital, Taunton, England.

Movement related brain macropotentials, electromyograms, electrodermal activity and the EKG were recorded from two age-separated groups each of six normal people and a group of six patients. All subjects were right handed. The age of the first group was $25.3 \pm 6.6$; the second group $53.8 \pm 13$. The third group consisted of untreated patients with Parkinson's Disease with a mean age $54.8 \pm 13$. The subjects carried out a self paced skilled task in which the sweep of an oscilloscope initiated by a left-hand press had to be terminated within $40-60 \mathrm{msec}$ by a right-hand press. Electromyograms were recorded from flexor and extensor muscles of each forearm. Scalp macropotentials were recorded from $F p_{2}, C_{2}, P_{2}$ and bilaterally from pre- and post-central locations, each electrode being referred to linked mastoids. Data processing was carried out by a PDP-12 computer. The Bereitschaftspotential and the motor cortex potential (Papakostopoulos, J. Physiol. 1978, pp72-73) in the young age group were significantly larger compared to the higher age group and the Parkinson patients. The quality of performance in terms of correct hits had a similar distribution. There was no significant difference between the Parkinson group and the comparable age group in terms of performance or of macropotentials although there was increased variability with the Parkinson group and some instances of suppression or abolition of the Bereitschaftspotential even though the post-action positivity associated with skilled performance was preserved. 


\section{Relevance of Exogenous and Endogenous Growth Hormone on the Levodopa Cerebral Effects}

P. Papavasiliou and F. H. McDowell. Department of Neurology, Cornell University Medical College, New York Hospital, New York, 10021, USA

To investigate further the role of $\mathrm{GH}$ on the therapeutic and side effects of levodopa, exogenous HGH was administered to four Parkinson patients with varying responses to levodopa therapy. 5 I.U. of $\mathrm{HGH}$ were administered on alternate days for four days and the effects of this hormone on the patients' symptomatic control, dyskinesia, plasma $\mathrm{GH}$ and dopa levels were evaluated and compared to those resulting from the saline injections. We have demonstrated that administration of exogenous $\mathrm{HGH}$, even during marked and sustained elevations of plasma $\mathrm{GH}$, does not alter the therapeutic or the side effects of chronic levodopa therapy. With one exception, plasma dopa levels after $\mathrm{HGH}$ remained unchanged when compared to controls. It is concluded that neither the endogenous increases of $\mathrm{GH}$ in response to levodopa, nor those attained following exogenous HGH modify in any consistent way the therapeutic and side effects of chronic levodopa therapy, and that the episodic release of $\mathrm{GH}$ in response to levodopa occur independently of its cerebral effects.

\section{Topographical Relationship Between Extrapyramidal and} Limbic Systems in the Basal Forebrain: A Comparative Study in the Rat, Cat and Monkey

A. Parent, Laboratoires de Neurobiologie, Faculté de Médecine, Université Laval, Québec, Canada

Extrapyramidal and limbic structures are known to overlap particularly in the ventral striatum-olfactory tubercle area and within the globus pallidus (GP)-substantia innominata region. The latter forebrain area was studied with regard to its projection towards the habenula by means of the horseradish peroxidase (HRP) method in the rat, cat and squirrel monkey. In the rat, the entopeduncular nucleus (EN) (the equivalent of the internal pallidum (IP) of primates) can be shown to be the major source of forebrain afferents to the lateral habenula (LH) (see also Herkenham and Nauta, 1977). In the cat, however, the largest collection of forebrain neurons projecting to the LH results in a labelling of numerous neurons located along the ventromedial border of the IP and within the internal medullary laminae. In contrast, only a few positive neurons could be found in the IP itself after LH injections. The distribution of these peripallidal HRPlabelled neurons appears strikingly similar to that of the strongly-stained acetylcholinesterase (AChE)-containing neurons surrounding the GP in primates. In all three species, these $\mathrm{AChE}$ neurons appear related more to the underlying substantia innominata than to the GP whose neurons are only weakly reactive for $\mathrm{AChE}$. These findings suggest that the organization of the extrapyramidal and limbic systems relationship at pallidal level varies greatly from rodents to primates. (Supported by grant MT-5781 of the Medical Research Council of Canada.)

\section{Pharmacology of Dopamine Receptors in Man}

J. D. Parkes, C. D. Marsden, P. Bédard and P. A. Price. University Dept. of Neurology, King's College Hospital and Institute of Psychiatry, London, England

The pharmacology of central dopamine receptors is poorly understood. Different kinds of dopamine receptor may exist analogous to $\alpha$ and $\beta$ adrenergic receptors in the peripheral nervous system. In a search for specific and selective dopamine stimulants and antagonists, we have studied the actions of amphetamine, piribedil, bromocriptine and levodopa, before and after dopamine-receptor blockade with metoclopramide, pimozide, oxiperomide and tiapride. These antagonists do not have radically different actions. Apart from differences in potency, they all block the endocrine, movement and behavioural responses to dopamine stimulants. However some results are surprising. With agonists, inhibition rather than increase of movement may accompany low plasma bromocriptine concentrations. In equipotent antiparkinsonian doses the rise in plasma growth hormone level is less with bromocriptine than with levodopa. With antagonists, tiapride and oxiperomide in critical concentration prevent dyskinesias due to levodopa and bromocriptine without blocking other actions. No antagonist prevents the fall in blood pressure with dopamine agonists. We conclude there is no definite pharmacological evidence in man for the existence of different dopamine receptor types in the brain, since the observed differences are probably due to drug actions on non-dopamine systems as well as on dopamine-receptor systems in the brain.

\section{Extra Neo-Striatal Dopaminergic Activity and Akinesia \\ J.C. Péchadre. Laboratoire d'Electroencéphalographie, Hôtel-Dieu, 63003 Clermont Ferrant, France}

The stereotaxic unilateral destruction of pallido-fibers in the monkey (group A) is followed by neglect of the contralateral limb, which gradually decreased. The injection of apomorphine is followed by a state of shamrage associated with sniffing and gnawing. The bilateral stereotaxic destruction of pallido-fibers in the same monkeys (group B) is followed by moderate akinesia which is sustained. The injection of apomorphine at the same dose does not produce any abnormal motor activity. In the monkeys of group B, repeated injections of reserpine result in akinesia which is dosedependent. A typical cataleptic state may even be obtained with larger doses. The injection of apomorphine counteracts catalepsy and the monkey resumes almost normal behavior. The existence of extraneostriatal (cortical) depaminergic systems which might play a role in motor control in monkey will be discussed. (Work done in the Laboratoires de neurolbiologie, Québec, Canada, and I.N.S.E.R.M., Hôpital Foch, Suresnes, France).

\section{Presentation D'Un Appareillage Stereotaxique A Applications Multiples} J. Pecker, J. M. Scarabin et B. Vallée, Rennes, France

Poster radiographique présentant un appareillage stéréotaxique basé sur un principe de fauteuil iso-centrique avec visée télémétrique de face et profil à une distance foyer-plaque de $5 \mathrm{~m}$. Contrôle télévisé permanent. Changement de film incorporé. Possibilité de clichés stéréoscopiques avec décalage d'angle. Applications multiples au repérage stéréotaxique en particulier dans le traitement des mouvements anormaux.

\section{Parkinsonism and Autoimmunity. Antibody Against Human} Sympathetic Ganglion Cell in Parkinson's Disease

A. Pouplard, J. Emile, F. Pouplard, D. Hurez, Laboratoire d'Immunologie, Service de Neurologie Centre Hospitalier Universitaire 49036 ANGERS, France

$60,7 \%$ of patients with Parkinson's disease were shown by indirect immunofluorescence to have in their serum an antibody which reacts specifically with human sympathetic neuronal ganglion cells.

Autonomic abnormalities are common in Parkinsonism and studies are in progress to try to find a clinical correlation mainly with orthostatic hypotension, however this finding raise many other questions one of the most important being: which role can play immunological factors in Parkinson's disease?

\section{Dystonia Musculorum Deformans}

Three Non-Jewish Patients Treated with L-Dopa, A. H. Rajput, Saskatoon, Sask. Canada

Dystonia musculorum deformans (DMD) is an uncommon disorder in non-Jewish Whites and is virtually unknown in Negros. There is no report on the effects of Levodopa in Negros suffering from this disorder.

Three females, two non-Jewish White who suffered from sporadic and the third, a Negro with dominantly inherited dystonia musculorum deformans are reported. Their symptoms began at ages 5,6 and 7 years respectively and by ages 8,15 and 27 years the functional disability was moderate, severe and moderate in each case. On a daily dose of Levodopa, $750 \mathrm{mgm} ., 1,500 \mathrm{mgm}$., and $3,000 \mathrm{mgm}$. respectively, the improvement began within two weeks in each case and reached the peak in 6 months. The improvement was remarkable in all three patients. The last evaluations performed two years, eight years and six years on treatment respectively reveal sustained improvement in all cases. The second patient had minor dyskinesias on Levodopa, which subsided with slight reduction in the dosage. The third patient suffered from episodes of mild depression and labile hypertension. The response to Levodopa and the side effects from the drug were not related to the average or above average intelligence. (Movie films of patients before and on treatment will be exhibited)

There are no clinical, biochemical, genetic or ethnic parameters to predict the response of DMD patients to different drugs. Therapeutic trial on a small dose of Levodopa (up to $3,000 \mathrm{mgm}$. per day), for two months is safe and advisable in all patients.

Dystonia Musculorum Deformans is a relatively uncommon neurological disorder and includes a variety of biochemical and pathological disorders. In spite of all efforts the frozen brain specimens from untreated dystonic patients for biochemical analysis, will not be available in large 
numbers in the near future. At this stage of our knowledge in this disorder a multicentre collaborative study, choosing common clinical, physiological and biochemical evaluations will perhaps be more valuable research alternative.

\section{GABAergic Neurons and Axon Terminals in the Extrapyramidal} System as Demonstrated by Immunocytochemistry

C.E. Ribak, J. E. Vaughn and E. Roberts. Department of Anatomy, University of California, Irvine, CA. 92717 and Division of Neurosciences, City of Hope Medical Center, Duarte, CA. USA 91010

Glutamic acid decarboxylase (GAD), the enzyme that synthesizes the neurotransmitter, GABA, was immunocytochemically localized in neurons and axon terminals of the rat striatum, pallidum, entopeduncular nucleus and substantia nigra. Since GAD has been localized in other brain regions to neurons which have been identified as GABAergic neurons, it is highly probable that the presence of GAD within neurons in the extrapyramidal system is diagnostic for those neurons which use GABA as a neurotransmitter. In our studies, GAD was localized to the somata of medium-sized spiny neurons in the striatum and to medium-sized neurons in the pallidum. In the striatum, a small proportion of axon terminals were GAD-positive and these formed mostly symmetric synaptic junctions. In contrast, most of the axon terminals in the pallidum, entopeduncular nucleus and substantia nigra were GAD-positive. These terminals extensively surrounded dendrites and somata and most commonly formed symmetric synaptic junctions.

The pattern formed by GAD-containing terminals with the dendrites and somata of neurons in the substantia nigra was altered following ipsilateral hemitransections of the striato- and pallidonigral pathways. A marked reduction of GAD-positive terminals occurred throughout the substantia nigra, but the ventral $1 / 3$ of the pars reticulata showed a normal pattern of GAD-positive ax on terminals. This decrease of GAD-containing terminals in the substantia nigra is consistent with results from biochemical studies which have indicated that the striato- and pallidonigral pathways are GABAergic. In addition, our results suggest that a brain region other than the striatum or the pallidum has a GABAergic projection to the ventral substantia nigra. Since autoradiographic tracing results have shown that the subthalamic nucleus has a projection limited to this ventral tier of the substantia nigra, it is likely that the subthalamonigral pathway uses GABA as a neurotransmitter.

Supported by USPHS grants NS12116 and NS1615 from the NINCDS.

\section{Regional CNS-GABA Receptor Binding and} GABA Levels in Parkinson's Disease

P. Riederer, H. Möhler, L. Boltzmann-Institute for Clin

Neurobiology, Lainz-Hospital A 1130 Vienna, Austria and

Pharmaceutical Research Dept., Hoffmann-LaRoche, Basle, Switzerland

Possible alterations of GABA receptor sites in Parkinson's disease were investigated by GABA recepter binding in several regions of Parkinsonian and control brains (frontal cerebral cortex, putamen, n.caudatus, hippocampus, n.accumbens,, pallidum, s.nigra).

In addition, an attempt has been undertaken to follow the post mortem changes of GABA in a control group. Regression analyses do not show any alterations of GABA-concentrations in different brain areas from 2 to 19 hours post mortem. In comparison with these results, in parkinson's disease there was a mild, but significant drop in GABA-levels which are discussed in relation to the rate of $\mathrm{GABA}$-synthesis. In contrast, depression syndrom did not change regional GABA-levels. However, preliminary data demonstrate a trend to increase GABA-levels in schizophrenia in lenticulate nuclei with normal levels in s.nigra, a fact which seems to be due to chronic neuroleptic treatment.

Responses of Brain Neurochemistry to Levodopa Therapy in Parkinson's Disease

U. K. Rinne, V.Sonninen and H. Laaksonen, Department of Neurology, University of Turku, Turku, Finland

Treatment with levodopa in combination with decarboxylase inhibitor increased dopamine turnover in the substantia nigra-striatum system more selectively than levodopa alone. Levodopa treatment did not have any significant effect on the concentration of noradrenaline, 5-HT or 5-HIAA in the parkinsonian brain. The activity of TH and DDC was decreased in the basal ganglia of the parkinsonian brain. Levodopa treatment did not have significant effect on activities of these enzymes. However, there was a negative correlation between the duration of levodopa treatment and $\mathrm{TH}$ activity. The activity of $\mathrm{AChE}$ was decreased in the parkinsonian extrapyramidal nuclei with the exception of pallidum. Levodopa treatment did not change the activity of AChE. The activity of GAD was decreased in the parkinsonian extrapyramidal nuclei but the content of GABA decreased only in the cerebral and cerebellar cortex. GABA receptor binding was significantly decreased in the substantia nigra of the parkinsonian brain. (Supported by grant from Medical Research Council, Academy of Finland.)

\section{Afferents to the Nucleus Subthalamicus}

E. Rinvik, I. Grofova, J. M. Deniau, J. Feger and C. le Guyader, Anatomical Institute, University of Oslo, Norway and Laboratoire de Physiologie des Centres Nerveu 4, place Jussieu, 75230 Paris, France

The horseradish peroxidase (HRP) tracing method has been used to analyse the afferent connections of the nucleus subthalamicus (STH) in the monkey (Macaca cynomolgus). In 4 cases the injections were limited to STH with only little spreading of HRP along the needle track in Forel's field $\mathrm{H} 2$. In the remaining cases HRP was deposited along the borders of STH and diffused to neighbouring structures. These cases served as useful controls. Following injections within STH the largest number of labeled cells is found in the lateral pallidal segment. Localization of labeled cells within this nucleus varies according to injection sites, thus confirming the topographical organization of the pallidosubthalamic fibers. Moreover, many labeled cells are found in the medial hypothalamus, both parts of the ipsilateral and, occasionally, also the contralateral substantia nigra including paranigral cell groups, in the nucleus dorsalis raphe, locus coeruleus and nucleus pedunculopontinus on both sides, and in the bed nucleus of the stria terminalis. Very few cells are seen in the substantia innominata, nucleus centralis amygdalae and cerebral cortex. No labeled cells are seen in the medial segment of the globus pallidus. Similar observations were made in several cats with iontophoretically ejected HRP in STH. A comparison with control cases in which HRP was mainly deposited outside STH suggests that nucleus raphe dorsalis, nucleus pedunculopontinus and the bed nucleus of stria terminalis project to STH in addition to the lateral pallidum. Whether nigrofugal and coeruleofugal fibers actually terminate in or only pass through STH could not be definitely settled in the present study.

\section{Clinical and Pharmacological Correlations During Fluctuations} Observed in the Long-Term Levodopa Therapy

P. Rondot, J. L. Ribadeau-Dumas and R. D. Ziegler, Service de Neurologie, Centre Hospitalier Sainte-Anne, Paris, France.

The efficacy of levodopa varied a great deal throughout the day after few years of treatment. This variation could be classified in four types according the time of levodopa administration or not: fluctuations occuring early or late after taking the drug, paroxystic fluctuations and circadian fluctuations. This fluctuation and the abnormal movements were correlated with the plasmatic levels of levodopa, prolactin and G.H. The effect of apomorphine was tested in a few patients. This study was performed in an attempt: - to determine the response of striatal dopamine receptor sites compared to the others in Parkinsonian treated for a few years. - to precise the pathophysiology of the fluctuation and abnormal movements: hyposensitivity or hypersensitivity of receptor sites, blocking of these receptors. The results tend to prove that the receptors, sometimes, become more sensitive to the variations of the levodopa level than to the highest levels. This suggests two different dopamine receptors.

\section{Treatment of Parkinson's Disease with a New L-DOPA Preparation Alone or in Combination with a New DDI (Fentiazac)}

S. Ruggieri, L. Conti, M. Baldassarre, A. Agnoli, P. Falaschi, A. Rocco, R. D'Urso, G. Frajese, - Clinica Neurologica dell'Università delL'Aquila. Italy. - Patologia Speciale Medica.

$$
\text { Università di Roma. Italy }
$$

The treatment of Parkinson disease presents some problems partially due to the therapy with L-Dopa-dopa decarbossila se inhibitor (DDI). In fact the "on-off" phenomenon, the freezing, sometimes the quick decrease in efficacy, the presence of involuntary movements, seem to be connected with the type of DDI used and with the standard Dopa/inhibitors ratio, in the associations commonly available in the commerce. 
These aspects can be also noted in the PRL response to the drugs. In fact it is possible to observe a different plasma PRL change for each DDI or for each association.

20 parkinsonian patients, divided in two groups, have been studied. In the first group the non responders subjects and the subjects with secondary neurological effects were collected. The second one is represented by untreated patients.

A new L-Dopa preparation, SEC-L-Dopa (Super Enteric Coated LDopa) was administered alone to all patients; when the highest clinical efficacy or when the daily dose of $5 \mathrm{~g}$ of L-Dopa were reached, a new DDI, Fentiazac (2-phenil-4-chlorophenylthiazol-5-ylacetic acid) was added in fixed dosage.

The dose of Dopa were reduced in case of improvement.

The therapeutic response to this therapy was as the classic one. A slight improvement in the parkinsonian symptomatology shows a good relationship between L-Dopa and Fentiazac. Anyway the neurological problems, like on-off and diskinesia, were not overcome by this new treatment.

In our view the main problem, in the standard treatment with L-DopaDDI, is the prefixed standard ratio.

The study of the tubero-inphundibular system might be an important tool for a better understanding of the parkinsonian symptomatology.

\section{L'Akinesie Parkinsonienne et L'inertie Motrice} Des Lesions Frontales. Ou Situer la Difference

O. Sabouraud, P. Hinault, H. Allain, P. Toulouse, R. Le Bars, Service de Neurologie, C.H.U. - RENNES - 35000 - FRANCE

La notion d'inertie motrice (I.M.) utilisée dans le cas des lésions des aires pré-motrices des lobes frontaux désigne-t-elle un phénomène semblable à l'akinésie parkinsonienne (A.P.) ou un phénomène différent? Cette question ne se pose guère pour la marche, la parole, l'écriture. Mais, dans les déplacements spontanés des membres (actogramme), la manipulation, les réponses manuelles à une commande, les descriptions se recouvrent. L'A.P. s'observe dans le contexte d'une impression subjective de difficulté de gène, de lourdeur, l'I.M. avec la tendance à la stéréotypie, à l'appauvrissement des séquences gestuelles, avec un défaut de programmation des tâches complexes. La mesure des temps de réaction, en faisant varier la complexité a) des stimuli (uni ou pluri-sensoriels) b) des réponses (presse-bouton ou trajectoire) contribuent à analyser les deux phénomènes. Deux paramètres physiologiques: a) le cycle d'excitabilité du réflexe $\mathrm{H}$; b) la variation contingente négative au niveau cortical sont étudiés (données préliminaires) dans leurs relations avec l'A.P. et l'I.M. selon l'hypothèse que les deux phénomènes intéressent des étapes différentes de la programmation motrice.

\section{Considerations About the ACH/DA/GABA Balance in the Striatum by the Hyperkinetic Syndrome Induced by d-Tubocurarine Intrastriatally Applied}

E. Scarnati, C. Forchetti, S. Ruggeri and A. Agoli, Depts of Physiology and of Neurology. School of Medicine - University - 67100 l'Aquila - Italy

It is known that the intrastriatal injection of d-Tubocurarine (dT) induces in the rat the appearance of an hyperkinetic syndrome characterized mainly by involuntary movements of the limbs and of the head. The mechanism underlying this syndrome is still unclear, being however imputable to a perturbance induced by $\mathrm{dT}$ on the striatal Dopamine/Acetylcholine/Gaba relationships. For these reasons we have tested the effects of the pretreatment with various drugs interfering with neurotransmitter systems infused via chronic cannulae exactly in the intrastriatal injection site of dT. The results show that drugs interfering with cholinergic transmission (Atropine, Nicotine, Carbachol, Metacholine and Physostigmine) do not modify the abnormal movements. Conversely, Haloperidol and Gaba itself prevented or reduced them. Likewise, the preventive depletion of the central cathecolamine levels by systemic alfa-metil-ptyrosine, reduces the choreiform movements. These data support the view that intrastriatal dT produces the abnormal movements through a dopaminergic action and point out the possibility of a direct Gaba modulation of the dopaminergic mechanism in the Striatum. The ineffectiveness of a such direct control may be implicated in the genesis of the involuntary movements appearing in human diseases such Huntington's Chorea in which a decrease of the intrastriatal Gaba levels is well documented.

\section{Control of Voluntary Ballistic and Ramp Movements} in Patients with Parkinson's Disease

B. T. Shahani, R. R. Young, Department of Clinical Neurophysiology, Massachusetts General Hospital and Harvard Medical School,

Fruit Street, Boston, MA 02114

Voluntary elbow flexion movements during the task of matching a step displacement of a line in an oscilloscope were studied in 32 normal subjects. 39 patients with Parkinson's disease and 9 patients with essential tremor (ET). Simultaneous surface EMG activity from a pair of antagonistic muscles (biceps and triceps), velocity of movements, reaction times (latency of initial EMG response in agonist after the visual command signal) and pattern of EMG activity were recorded during fast "ballistic" as well as smooth "ramp" movements. Duration of various components (Ag 1 -initial burst in agonist: An 1 - activity in the antagonist during electrical silence after $\mathrm{Ag} \mathrm{I}$; $\mathrm{Ag} 2$ - second burst in agonist), which constitute the normal triphasic EMG pattern during "ballistic" movements, were within normal limits in all patients. In patients with Parkinson's disease, values for reaction time $(267 \pm 83 \mathrm{msec})$ and velocity $(96 \mathrm{~cm} / \mathrm{sec} \pm 47)$ were both significantly altered $(\mathrm{p}<.001)$. During both "ballistic" and "ramp" movements, there was a tendency for EMG activity to be decomposed into discrete bursts which had a frequency similar to the predominant frequency of their action tremor in patients with ET and 1-3 Hz faster than the "tremor-at-rest" frequency in patients with Parkinson's disease. It is concluded that both fast "ballistic" and slow "ramp" movements are affected in Parkinson's disease. Furthermore, the tremor-at-rest of Parkinson's disease is replaced by a different kind of tremor during voluntary activity unlike the tremor activity (which may reflect enhancement of underlying action tremor) during similar tasks seen in patients with ET.

Comparison of Various New Drugs in the Management of Dyskinesias J.Siegfried, Neurochirurgische Klinik, Universitätsspital, $\mathrm{CH}-8091$ Zürich, Switzerland

A number of major problems in management of patients on levodopa therapy remain. Dyskinesias, particularly the one of the "on-off" reactions, are still difficult to treat and different therapeutical approaches have been made. The use of very small dosage of levodopa in combination with peripheral decarboxylase inhibitor (Madopar 62.5) can temporary improve markedly the condition and the iatrogenic dyskinesias, but involuntary movements may eventually return later on. Dopaminergic agonists, alone or better in combination with levodopa therapy, are sometimes useful and our experience rests on bromocriptine, lergotrile, pyridil, as well as Atrium, budipine, diprobutine and mazindol. Some encouraging observations will be reported. Besides the conservative methods, the author has a favorable experience with the stereotactic procedures which are today safe and very effective, as far as dyskinesias are concerned.

Anterior Cerebellum and Catecholaminergic Nuclei:

Anatomical and Biochemical Evidence for Direct Interconnections

S. R. Snider, R. S. Snider and S. Fahn, Dept. Neurology, Columbia University, New York, 10032, and Center for Brain Research, U. Rochester, NY 14642

The overlap in symptomatology of cerebellar and "extrapyramidal" diseases is usually attributed to functional interaction in ventrolateral thalamus and other areas to which both systems project. We have evidence from studies in rat, cat and monkey that the cerebellum may directly influence catecholaminergic nuclei related to the "extrapyramidal" system. Silver-stained preparations 1 to 8 weeks after cerebellar lesion showed degenerating nerve terminals in ventral tegmental area (VTA), substantia nigra (SN) and nuclei locus ceruleus (LC) and subceruleus. Noradrenergic fibers of LC project directly back to cerebellum, while the dopamine (DA)containing neurons of VTA and SN may be part of a cerebellar-tegmentalstriatal-cerebellar circuit that participates in neural control of posture and muscle tone.

In rat, reduction of cerebral DA concentration and turnover resulted from surgical lesion of deep cerebellar nuclei, and increase of cerebral DA resulted from anterior cerebellar cortex lesions made surgically or by injection of kainic acid.

We conclude that the anterior cerebellum may be an important source of input to the catecholaminergic nuclei in the brainstem. 
Biochemical and Pharmacological Tests of Central Monoamine Functions in Various Dyskinesias

T. L. Sourkes and S. Lal, McGill University, Montreal, Canada H3A IA I

The authors propose to review the use of the probenecid test (in relation to monoamine metabolites of the CSF) and simple pharmacological tests for the investigation and treatment of movement disorders other than Parkinson's disease. New data in regard to spasmodic torticollis will be presented.

Investigations Concerning Motor Control in Man Under Hypotonia of Central and Peripheral Origin

A.Struppler, H. Altmann, F. Erbel, F. Velho, Neurologische Klinik der Technischen Universität München. F.R.G.

Motor control in Parkinsonian patients was investigated before and after stereotaxic thalamotomy of one side and compared to the non-operated side. In normal subjects motor control before and during the stage of differential block was studied and compared with the findings in operated Parkinsonian patients. Two identical torque motors were used to deliver simultaneously torques to both elbow joints. EMG was recorded from brachialis and triceps muscles on both sides together with the angular displacement. Following stereoencephalotomy, maximal force and tendon reflexes remained unchanged. Load compensation to a sudden stretch was impaired on the operated side, the angular displacement was increased, the first components of the stretch reflex were decreased, the following volitional EMG activity increased. Load compensation during hypotonia induced by differential nerve block in normals showed similar changes.

\section{Tremor and Response to L-DOPA Treatment}

Alvar Svanborg, Department of Geriatric and Long Term Care Medicine, Vasa Hospital, University of Göteborg, Göteborg, Sweden.

Our material of 134 parkinsonian patients, treated with L-dopa since 1968-70 has been repeatedly analysed concerning possible relationships between the outcome of the treatment and age at the start of the disease, at the start of treatment, as well as symptomatology and severity of handicaps. The improvement in ADL-disability during L-dopa treatment has been found to be directly correlated to the occurrence of tremor before treatment but universely correlated to age. The occurence of parkinsonian tremor before treatment was, however, not correlated to age, nor was the severity of the parkinsonian symptoms. The improvement of tremor, but not of the other symptoms, was negatively correlated to the duration of the disease. The appearance of muscular hypotonia was also negatively correlated to age but the appearance of mental confusion positively correlated to age. Improvement of speech disturbances was negatively correlated to age.

Our results show that the age of the patient and the existance of tremor or not in the syndrome are the most important predictors of the therapeutic response.

Interconnections Between Striatum and

\section{Substantia Nigra in the Cat and Monkey}

J.Szabo, Department of Anatomy,

Faculty of Medicine, Ottawa, Ontario, Canada. KIN 9A9

Horseradish peroxidase studies in cats and monkeys revealed that the localization of striatal projecting cells in the substantia nigra is arranged in a mediolateral, anteroposterior and a dorsoventral topographical pattern. The feline retorubral nucleus appears to be an extension of the pars compacta. Neurons in the ventral tegmental area (VTA), the rostral and central linear nuclei (LR,LC), the interfascicular (IF), annular (Ann) and dorsal raphe (DR) nuclei also project to the striatum. The paranigral cell groups and the raphe system are more intimately related to ventral striatum and $\mathrm{n}$. accumbens (Acb). The topography of the striatonigral projections, as seen earlier with silver impregnation methods, was also verified. The majority of projection cells in the striatum were of medium size (appr $20 \mu \mathrm{m}$ ) with scanty cytoplasm. One or possibly•two more types of cell, heavily labeled were observed, the largest having a diameter of $30-35 \mu \mathrm{m}$. The EM findings are in good accord with these observations. (Supported by the Medical Research Council of Canada).
The Stereotaxic Target for Dyskinesias

R. R. Tasker, L. W. Organ and P. Hawrylyshyn, University Wing, Toronto General Hosp., Toronto, Ont., Canada. M5G IL7

Stereotaxic surgery remains an important means of treating dyskinesias. Using a computer graphics program we have reviewed the lesion sites made in patients with Parkinsonism, essential tremor, cerebellar tremor, dystonia musculorum deformans and dystonia associated with cerebral palsy out of a series of 198 procedures studied in detail. The target site appears identical for all. A computerized graphic physiological study of that target site using $100 \mathrm{HZ}$ stimulation demonstrates its location just rostral to the lemniscal relay for the lips and hand, caudal and medial to corticospinal primary motor fibres. Within the target zone can be identified the thala mic relay of a rostral vestibulo-thalamic pathway, and an area where stimulation induces hallucination of movement of parts of the contralateral body, modifies contralateral dyskinesia, and produces motor effects distinct from the dyskinesias themselves as well as from the tetanizing responses elicited in the primary corticospinal motor pathway. The significance of these observations with respect to brainstem organization and mechanisms of dyskinesia will be discussed.

\section{Altered Responses of Motor Cortical Neurons} as One Basis for Phenothiazine Rigidity

W. G. Tatton, P. Bawa and I.C. Bruce, Playfair Neuroscience Unit, University of Toronto, Toronto, Ontario. M5T 2S8

Compared to normal subjects rigid Parkinsonian humans show increased reflex EMG activity at a latency corresponding to that of the normal "M2" response in muscles stretched by imposed joint displacements (Tatton and Lee 1975). They also have a decreased ability to modulate the activity occurring over the "M2" interval according to "voluntary set". Further, antagonist muscles shortened by the imposed displacements show an inappropriate increase rather than the normal reciprocal decrease in EMG activity that is usually evident over the "M2" interval (Mortimer et al 1976). A distinct population of monkey motor cortical neurons (MCNs) show responses that are appropriately-timed in onset, peak time and duration to contribute to EMG activity in the stretched muscles during the M2 interval (based upon microstimulation and antidromic studies). About $50 \%$ of those neurons show reciprocal decreases in firing over a corresponding interval in response to displacements stretching the antagonist muscles. Monkeys chronically intoxicated with a phenothiazine tranquilizer (Atravet) show cogwheel rigidity and bradykinesia similar to Parkinsonian humans. The EMG responses to imposed wrist displacements are markedly increased beginning at the "M2" latency as compared to those in the same monkeys prior to and following the intoxication. All of the MCN's recorded during the intoxication responded with symmetrical increases in firing to imposed displacements stretching either the wrist flexors or extensors. Despite the loss of reciprocity in the responses of the MCN's during the intoxication, the appropriate timing with respect to the increased duration of the EMG responses was maintained. Based upon the assumption that the motor cortex contributes to the "M2" EMG activity and mediates the abnormal responses in phenothiazine rigidity, a tenative model for the altered central mechanisms underlying Parkinsonian rigidity will be presented.

\section{Degeneration of Targets Deprived of Catecholaminergic Innervation}

V. M. Tennyson, M. Budininkas-Schoenebeck, and J. Martin,

Depts. Anatomy, Pathology (Neuropathology), and Neurology, Columbia, College of Physicians \& Surgeons, N.Y

Subcutaneous administration of reserpine $(0.06-0.13 \mathrm{mg} /$ day) to pregnant Dutch rabbits from gestation days 14 through 22 (term at 30 days) almost completely depleted fluorescence in the maternal neostriatum. Fluorescence was absent in the fetal putamen when sacrificed 4 hours after the last drug injection, whereas control fetuses had many fluorescent profiles of axons and growth cones throughout this region. When fetuses were allowed to survive 6 days after the last dose of reserpine, some scattered fluorescent terminals were present in the putamen, but there were many fewer than in controls. Controls have islands of diffuse fluorescence and abundant fluorescent terminals. Electron microscopic examination of the fetal putamen after the last dose of reserpine (fetal days 21 or 22) or after the 6 day survival period (fetal day 27) revealed evidence of degenerative 
phenomena. There were degenerating multivesicular bodies and heterogeneous bodies. There were also cytoplasmic masses of whorled membranes and clumped filamentous or extremely dense amorphous material. Some of these degenerating profiles were in cell bodies and in processes that appeared to be dendrites. (Supported by Grant No. NS-11870, the United Cerebral Palsy Research \& Educational Foundation and the Parkinson's Disease Foundation).

Triggering and Resetting of Parkinsonian Tremor by Arm Displacement H. Teräväinen, D. B. Calne and E. V. Evarts, Lab. Neurophysiology, NIMH and Experimental Therapeutics Branch, NINCDS, Bethesda, Maryland, U.S.A. 20014

To determine the extent to which resting parkinsonian tremor is influenced by peripheral factors, single perturbations of $50 \mathrm{msec}$ duration were delivered to flex or extend the wrist at known tremor phases, either assisting or opposing ongoing tremor movements. The position of the wrist, and EMG of wrist extensor and flexor muscles were recorded and subsequently analyzed by computer.

It was found that the phase of tremor could be reset by suitably placed short duration perturbations (see figure). Furthermore, displacements delivered during absence of significant tremor could trigger tremor whose phase depended on the direction of the displacement. Results obtained thus indicate the presence of powerful peripheral proprioceptive tremor modulation in the displaced extremity. Such perturbations did not reset or initiate tremor in the contralateral limb, however. This was to be expected, since tremor in the two extremities was often asynchronous and of different frequency.

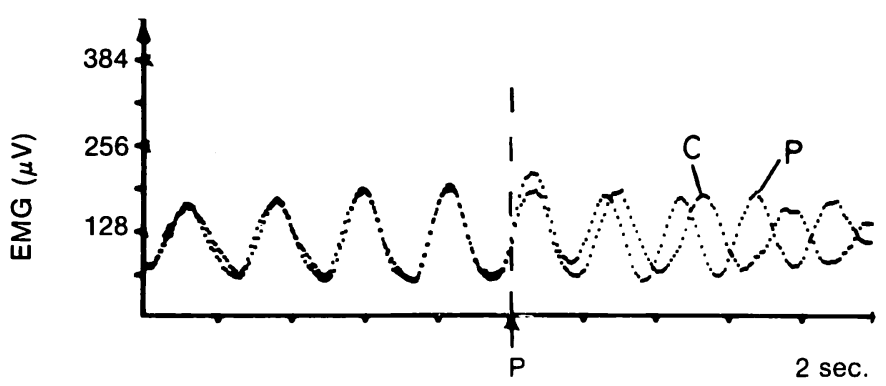

The figure illustrates resetting of tremor EMG activity (rectified, in the extensor muscles of the perturbed forearm. Eleven control trials without perturbation $(C)$ are superimposed on eleven trials with a perturbation $(P)$ which opposed extension of the wrist at the same tremor phase. $P=$ instant of perturbation.

Types of Dense-Core Vesicles and Their Relations to Mitochondria and to Clear Vesicles in Aplysia Californica

J.P. Tremblay and M.Colonnier, Laboratoires de neurobiologie, Hôpital de l'Enfant-Jésus, Québec, Canada. G1J 5B3

In the abdominal ganglion of Aplysia, dense-core vesicle populations establish different relationships with other organelles in ways which often appear as a caricature of what is only suggested in mammals and give interesting clues as to their possible functions. A large number are characterized by the fact that they do not agglomerate towards differentiated membranes. This is obvious for populations of very large granules completely filling groups of contiguous profiles which never contact each other. They are interpreted as neurosecretory. In other profiles, small dense-core vesicles surround mitochondria and patches of glycogen. They are often disposed as peripheral halos around groups of clear vesicles agglomerating towards differentiated membranes. It is proposed that they do not contain neurotransmitters but are related to mitochondrial activities such as storage of ATP, or movement of calcium ions. Other terminals contain only dense-core vesicles which do agglomerate towards differentiated membranes, suggesting that they contain transmitters liberated at specific postsynaptic sites. Finally, some terminals contain clear and densecore vesicles both abutting on differentiated membranes. This suggests the release of two different transmitters, or of a modulator or neurohormone with a transmitter, by a single terminal.

\author{
Role of the Globus Pallidus in the Initiation \\ and Execution of a Goal-Directed Movement \\ E. Trouche, D. Beaubaton, G. Amato, A. Grangetto, \\ Institut de Neurophysiologie et Psychophysiologie \\ C.N.R.S. Marseille, France
}

Human pathology and experimental studies in animals have show that lesions of the basal ganglia produce severe disturbances of motor activity and posture. Few data exist about the role of these nuclei in the control of the different phases of a motor act. An experiment has been performed to investigate the effects of temporary or permanent exclusion of the pallidum on the initiation and execution of a visually guided movement. In baboons, trained to perform a pointing movement, reaction times, movement times and spatial precision of pointing have been studied. The decreasing of reaction times following pallidal exclusion stresses the importance of this structure in the initiation of movement. Moreover, the observed impairments concerning movement times and precision of pointing emphasize also the participation of the pallidum in the execution of a goal-directed movement. (Supported by INSERM grant 29-76-61).

Neuroendocrine Modulation in Tardive Dyskinesia

A. Villeneuve, Research Director, Dept. of Psychiatry, Hôpital de l'Enfant-Jésus, Quebec; Clinical Research Unit, Centre Hospitalier Robert Giffard, Beauport; Professor, Dept. of Psychiatry, Faculty of Medicine, Laval University, Quebec

The functioning of the extrapyramidal system reveals itself increasingly complex. In addition to dopamine and acetylcholine, substances like GABA and enkephalin also appear to be involved in its regulation. Moreover, there is now growing evidence that chemical synaptic transmission can be influenced by hormones and the beneficial effect of estrogens on L-DOPA-induced dyskinesias and on tardive dyskinesia has been reported lately. Estrogens seem to interfere with dopaminergic transmission in the striopallidal system. This relationship between dopamine and estrogens is interesting as the presence of catechol estrogens, compounds that might play an important role in neuroendocrine regulation, has been demonstrated in some areas of rat brain. Clinical data concerning the therapeutic action of estrogens on tardive dyskinesia are presented and the theoretical implications discussed.

Lergotrile in the Treatment of Parkinson's Disease

W. J. Weiner, P. A. Nausieda, C. Goetz and H. L. Klawans, Department of Neurological Sciences, Rush Presbyterian St. Luke's Medical Center, Chicago, Illinois 60612. P. Volkman, Department of Pediatrics, Loyola Medical Center, Maywood, Illinois

Lergotrile mesylate, a direct-acting dopamine receptor agonist, was administered for up to 10 months to 25 patients with Parkinson disease. Six of these patients were not concurrently receiving levodopa. Five of these six patients who were not on levodopa showed definite improvement in parkinsonian signs and symptoms on lergotrile. These results indicate that lergotrile is efficacious in the treatment of Parkinson disease, independent of any interaction with levodopa. Nineteen other patients who received lergotrile were experiencing long term side effects of levodopa therapy. We found that lergotrile was of benefit in the treatment of the on-off phenomenon but that it had little or no effect on increasing bradykinesia and tremor. In addition lergotrile could potentiate levodopa induced dyskinesia. In $40 \%$ of our patients lergotrile induced a chemical hepatitis. Liver biopsy revealed evidence of an acute non specific hepatitis. All liver function abnormalities returned to normal after withdrawal of the drug. This high degree of hepatotoxicity associated with the clinical use of lergotrile will preclude its clinical use.

\section{Low CSF Tetrahydrobiopterin Levels in Parkinsonism}

A.C. Williams, R. Levine, D. Robinson, W. Lovenberg and D. B. Calne, National Institutes of Health, Bethesda, Maryland U.S.A. 20014

The availability of tetrahydrobiopterin, the natural cofactor for tyrosine and tryptophan hydroxylase, is of critical importance for the synthesis of dopamine, serotonin, and norepinephrine. Tetrahydrobiopterin levels have therefore been measured in the CSF of unmedicated patients with Parkinson's disease and in normal controls. Levels decreased with age in the normal controls $(r=.48)$. Patients with Parkinson's disease had lower levels $($ mean $=8.93 \mathrm{pmol} / \mathrm{ml}, \mathrm{S}$. E.M. $=.95, \mathrm{n}=10)$ than age matched controls 
$($ mean $=17.7 \mathrm{pmol} / \mathrm{ml}$, S.E.M. $=1.67, \mathrm{n}=10$ ). This was significant at the $\mathrm{p}=<.001$ level. Levels were also low in patients with Progressive Supranuclear Palsy (mean $=8.5 \mathrm{pmol} / \mathrm{ml}$, S.E.M. $=1.1, \mathrm{n}=7$ ) and the Shy-Drager syndrome (mean $=3.4 \mathrm{pmol} / \mathrm{ml}$, S.E.M. $=1.1, \mathrm{n}=3$ ) but were normal in focal dystonia (mean $=17.3 \mathrm{pmol} / \mathrm{ml}$, S.E. $M .=2.6, \mathrm{n}=7$ ).

It is likely that these low levels of tetrahydrobiopterin are a reflection of loss of dopaminergic neurons. These results suggest that increasing tetrahydrobiopterin activity might have a therapeutic action in parkinsonism.

\section{Affective-Motivational Deficits Underly Changes in Lever Pressing After Dopamine Blockade}

Roy A. Wise, Psychology Dept., Concordia University, Montreal, Quebec, Canada H3G 1 M8

Lever pressing and alley running for food, amhetamine, cocaine and brain stimulation rewards were challenged with pimozide at doses of up to $1.0 \mathrm{mg} / \mathrm{kg}$ in rats. Ability to initiate and carry out well-learned habits were not impaired as shown in initial tests and initial portions of repeated tests. In each case, however, responding decreased within and across sessions with the higher doses. The response decrements paralleled decrements caused by reducing or eliminating the reward payoff in non-drugged animals. In the case of amphetamine reward and cocaine reward the animals increased their responding to compensate for the lower doses of pimozide. Our data rule out the view that these doses of pimozide simply or seriously impair the motor responsiveness of the animal. Rather they fit well with the notion that pimozide has as its most interesting and lowest dose effects the attenuation of those qualities of food, amphetamine and brain stimulation which give these stimuli their normal rewarding impact. It is not clear whether the motivational deficits reflected in our data result from alterations in striatal or rather extrastriatal dopamine function.

\section{Can Compartmentation of $\boldsymbol{\gamma}$-Aminobutyric Acid (GABA) Metabolism be Used to Advantage in the Therapeutic Regimens?}

J.D. Wood, Department of Biochemistry, University of Saskatchewan, Saskatoon, Sask. Canada S7N 0W0

When a neurological disease involves an abnormal metabolism or function of a neurotransmitter substance, an obvious therapeutic approach is to administer drugs which counteract these disease-related changes. Unfortunately, when the abnormality is confined to specific regions of the brain, the drugs are equally likely to affect the metabolism and function of the neurotransmitter in the still-normal areas of the nervous system, thereby leading to the possibility of undesirable side effects.

On the other hand, metabolism of the neurotransmitter, GABA, is compartmented in brain tissue, being located in glia, in neuronal perikarya and in nerve endings. Moreover, the loss of neurons or the occurrence of gliosis, events that are known to occur in neurological diseases including Parkinsonism, result in a change in the ratio of glia to neurons. Thus, the possibility exists that this change in ratio may be exploited for therapeutic purposes by using drugs which affect GABA metabolism specifically in glial cells. In this case, the drug induced changes will be emphasised in diseased areas of the brain with lesser effects occurring in normal areas of the nervous system. Little is known about the relative contribution of nerve endings, glia and neuronal perikarya to the control of the GABA concentration in the synaptic cleft, yet this information is vital if a rational approach is to be taken towards the type of drug therapy suggested above which is aimed specifically at diseased areas of the nervous system. A vigorous research effort therefore seems warranted in an attempt to unravel the intricacies of the control mechanisms for synaptic cleft GABA levels.

\section{Drug Induced Involuntary Movements - Consideration of} its Pathophysiology and Treatment

Melvin D. Yahr, M. D., Department of Neurology,

Mt. Sinai School of Medicine, University of the City of New York, New York, N.Y. 10029

Hyperactivity of the striatal dopaminergic system has been implicated as producing the pathophysiologic state underlying hyperkinetic and dyskinetic movement disorders. Attempts to utilize drugs which reduce dopaminergic function have to date given less than fully satisfactory results in controlling such conditions. The reciprocal relationship between dopaminergic and cholinergic function has promoted study of agents capable of increasing the activity of the latter and hence, re-establishing homeostasis of these neurotransmitters. Recent studies utilizing orally administered choline chloride have shown its ability to significantly elevate cerebrospinal fluid ACH and presumably brain levels as well. The results of clinical trials of this agent in movement disorders both drug induced and those occurring de novo, will be presented. The differing effects obtained are analyzed in respect to the nature of transmitter dysfunction underlying these varying movement disorders. (Supported by Grants N.I.H. NS11631-05 and the Parkinson's Disease Foundation. N.Y.

\section{Functional Activity of Monoamine Oxidase in L-DOPA Therapy}

M. B. H. Youdim and P. Riederer, Faculty of Medicine, Technion, Haifa, Israel and Ludwig Boltzmann Institute for Neurochemistry, Vienna, Austria

In rats tranylcypromine (TCP) $(20 \mathrm{mg} / \mathrm{kg})$ administered totally inhibited brain monoamine oxidase (MAO) oxidation of dopamine (DA). When LDopa $(50 \mathrm{mg} / \mathrm{kg})$ was given $30 \mathrm{~min}$. after the TCP, brain DA and noradrenaline (NA) concentration rose markedly and the rats displayed characteristic behavioral changes and locomotor activity. When L-Dopa $(50 \mathrm{mg} / \mathrm{kg}$ ) was given at $12 \mathrm{~h}$ and $18 \mathrm{~h}$ after TCP the characteristic hyperactivity syndrome appeared only at $12 \mathrm{~h}$. Also, brain DA and NA markedly increased only at $12 \mathrm{~h}$. Inhibition of MAO oxidation of DA was less than $85 \% 18 \mathrm{~h}$ after TCP. The hyperactivity syndrome observed with TCP and L-Dopa could not be established with L-Dopa following the selective inhibitors of MAO 'type $A^{\prime}$ ' and 'type $B$ ', namely clorgyline and deprenil respectively. However, at low doses $(5 \mathrm{mg} / \mathrm{kg})$, these inhibitors in combination can elicit hyperactivity syndrome in rats receiving L-Dopa. It is concluded that in the rat brain DA is deaminated by 'type A, and 'type B, $\mathrm{MAO}$ in vivo and it is only when both forms of the oxidase are inhibited by more than $85 \%$ that there is an increase in Da functional activity, illustrated by the onset of hyperactivity syndrome. Recently we have shown that deprenil, a selective MAO 'type B' inhibitor without "cheese effect" can potentiate the therapeutic effect of L-Dopa and is also effective in 'on-off' disabilities with end of dose akinesia in Parkinsonian patients. Post mortem studies on brains from Parkinsonian patients who responded to 10 $\mathrm{mg}$ daily dose of deprenil have indicated that MAO activity is inhibited by more than $90 \%$. These results will be discussed in relation to the clinical efficacy of deprenil inhibition of brain MAO activity.

\section{A Quantitative Analysis of Single Motor Unit}

Discharge Patterns in Patients with Parkinson's Disease

R. R. Young, B.T.Shahani, Department of Clinical Neurophysiology, Massachusetts General Hospital and Harvard Medical School. Fruit Street, Boston, MA 02114

Microneurographic data are now available from studies of Hagbarth and colleagues, which permit detailed analysis of the behavior of the spinal stretch reflex (including muscle spindle afferent input) in the 3 to $7 \mathrm{~Hz}$ tremor-at-rest of Parkinson's Disease and in a type of physiological tremor enhanced by the superimposition of passive 3 to $7 \mathrm{~Hz}$ sinusoidal stretch upon a tonically contracting muscle in normal subjects. The extrafusal muscle contractions in the tremor-at-rest are organized according to the principle of alpha-gamma coactivation. This is also true of voluntary alternating tremor-like contractions but two discrete bursts of spindle afferent activity are not seen in association with the en hanced physiological tremor which appears to result from modulation only of alpha montoneurons by segmental stretch reflex mechanisms. An analysis of single motor unit behavior has demonstrated orderly recruitment from smaller to larger units in each burst of tremor-at-rest, voluntary tremor and enhanced physiological tremor. On the other hand, units do not appear to be recruited anew in each burst of essential-familial tremor. In these latter tremors, the tonic background EMG activity appears to be interrupted by rhythmic tremulous pauses in activity. This data is compatible with the hypothesis that the tremor-at-rest of Parkinson's Disease is a response to rhythmic volleys descending from supraspinal centers and acting upon segmental spinal structures in an apparently normal way with alphagamma coactivation and orderly recruitment in each EMG burst. Stretch reflex mechanisms in these patients also appear to be normally responsive to peripheral perturbations which can produce marked changes in amplitude and phase of the ongoing tremor. With repeated stretch, the newly reset tremor-at-rest can be time-locked to the stimulus. These changes must occur both at the local spinal level and at supraspinal levels. 


\section{The Influence of Anti-Parkinsonian Drugs on Tryptophan Metabolism}

S. N. Young*, S. G. Gauthier ${ }^{+}$, G. M. Anderson\# and W.C. Purdy\#, *Department of Psychiatry, McGill University,

1033 Pine Avenue West, Montreal H3A 1A1, 'Department of Neurology and Neurosurgery, McGill University, \#Department of Chemistry, McGill University

Peripheral decarboxylase inhibitors which are now used in conjunction with DOPA in the treatment of Parkinsonism are capable of influencing brain biogenic amine metabolism by two mechanisms. First, 5-hydroxytryptophan (5HTP) formed in the intestine will not be decarboxylated to serotonin and will spill out into the plasma. We have found higher concentrations of 5HTP in the plasms of Parkinsonian patients recelving decarboxylase inhibitors than in those receiving DOPA alone. The concentration is small but might potentiate brain 5HT synthesis. Second, we have shown that peripheral decarboxylase inhibitors can decrease rat CSF indoleacetic acid (IAA). As CSF IAA seems to reflect CNS tryptamine turnover this suggests that tryptamine formed peripherally can cross the blood-brain barrier and that reduced plasma levels of tryptamine after decarboxylase inhibition may alter central tryptamine turnover. Thus peripheral decarboxylase inhibitors may decrease turnover of trace amines and potentiate turnover of 5HT in the CNS. (Supported by the MRC of Canada).
Experimental Tremor in Monkey: Activity of Thalamic and Precentral Cortical Neurons in the Absence of peripheral Feedback

Y.Lamarre and A.J. Joffroy.

Centre de recherche en sciences neurologiques, Département de physiologie, Université de Montréal, Montréal, Québec, Canada. H3C 3T8.

Single neuron activity was recorded in the sensorimotor cortex and in the thalamus of monkeys with experimental Parkinson-like tremor. The tremor was produced by electrolytic lesions of the ventromedial midbrain tegmentum. In order to eliminate sensory rhythmic impulses from the trembling limb, we recorded in curarized and unanesthetized animal and in animals after deafferentation of the trembling limb by section of the dorsal roots $\left(\mathrm{C}_{2}-\mathrm{T}_{4}\right)$.

In the curarized animal, rhythmic activity at the tremor frequency was recorded in the precentral motor cortex and in the lateral ventral portion of the thalamus. In the deafferented animals, rhythmic activity synchronous with the contralateral tremor was recorded in the precentral cortex in an area corresponding to the deafferented trembling limb. Rhythmic activity was also recorded in the lateral vestibular nucleus and in the bulbar reticular formation.

These results support the hypothesis that this experimental Parkinsonlike tremor in monkeys is generated by a central mechanism involving at least the thalamus, the motor cortex, and some vestibulo-and reticulospinal pathways.

(Supported by the Medical Research Council of Canada) 